\title{
1 A role for liquid-liquid phase separation in ESCRT-mediated nuclear envelope
}

\section{reformation}

${ }^{1}$ Department of Biochemistry and Biophysics, University of California, San Francisco, CA, USA

$6 \quad{ }^{2}$ Department of Oncological Sciences, Huntsman Cancer Institute, University of Utah, Salt Lake

7 City, UT, USA

$8{ }^{3}$ Department of Pharmaceutical Chemistry, University of California, San Francisco, San

9 Francisco, CA, USA

$10{ }^{4}$ Faculty of Chemistry and Pharmacy, University of Freiburg, Freiburg, Germany

$11{ }^{5}$ Chan Zuckerberg Biohub, San Francisco, CA, USA

$12{ }^{6}$ Quantitative Biosciences Institute, University of California, San Francisco, CA USA

$14 \dagger$ A.v.A., D.L., and I.E.J. contributed equally to this work

$16 *$ Correspondence to: Email: adam.frost@ucsf.edu, or katharine.ullman@ hci.utah.edu. 
At mitotic exit, microtubule arrays are dismantled in concert with the reformation of the nuclear envelope. We show how the inner nuclear membrane protein, LEM2, exploits liquid-liquid phase separation to direct microtubule remodeling and nuclear envelope sealing via the Endosomal

21 Sorting Complexes Required for Transport (ESCRT) pathway. LEM2 tethers membrane to

22 chromatin disks through direct binding between its LEM motif and the chromatin-associated

23 barrier-to-autointegration factor (BAF). Concurrently, a low-complexity domain within LEM2

24 undergoes liquid-liquid phase separation to coat spindle microtubule bundles. Spatially restricted,

25 LEM2's winged helix (WH) domain activates the ESCRT-II/ESCRT-III hybrid protein, CHMP7.

26 Together LEM2 and CHMP7 copolymerize around microtubule bundles to form a molecular "O-

27 ring" that promotes nuclear compartmentalization and initiates downstream ESCRT factor recruitment. These results demonstrate how multivalent interactions of a transmembrane protein, including those that mediate phase separation, coordinate localized ESCRT polymerization, mitotic spindle disassembly, and membrane fusion. Defects in this pathway compromise spindle disassembly, nuclear integrity, and genome stability. 
32 At the onset of "open" mitosis in mammalian cells, the nuclear envelope (NE) dissociates from the chromatin surface to allow for chromosome singularization, which enables the mitotic spindle to

34 segregate chromosomes into emerging daughter cells ${ }^{1}$. In parallel, mitotic phosphorylation events disrupt the affinity of inner nuclear membrane (INM) proteins for the chromatin surface, allowing them to recede, along with NE membranes, into the contiguous endoplasmic reticulum ${ }^{2}$. Upon mitotic exit during anaphase, a program of phosphatases reverses this process: INM proteins within the endoplasmic reticulum membrane regain affinity for DNA or DNA-associated partners to coat each mass of condensed chromosomes, referred to as a chromatin disk, with membranes ${ }^{3}$. DNA in a meshwork that restricts membrane access to the surface of the disk and guides the formation of minutes ${ }^{4,5}$. As the nascent nuclear envelope engulfs the chromatin disk, a subset of the nuclear membrane proteome transiently segregates into a distinct core region that coincides with microtubules emanating from centrosomes on the outer face of the disk and with central spindle microtubules on the inner face of the disk ${ }^{4,6-8}$. Of the INM proteins that enrich at the core, several belong to the LEM (Lap2-EmerinMAN1) domain family of proteins, which are characterized by their interactions with the nuclear lamina and, with respect to Lap2 and Emerin, their ability to bind to BAF via a conserved LEM-domain ${ }^{9}$. Recent studies identified another LEM family protein, the two pass transmembrane protein LEM $2^{10}$, as the sitespecific adaptor for the ESCRT machinery ${ }^{11,12}$. The ESCRT pathway ultimately coordinates spindle clearance and nuclear envelope fusion for the reforming nucleus ${ }^{6,13}$.

52 Within the core region of reforming nuclei, spindle disassembly and nuclear envelope (NE) fusion occur coordinately at sites of envelope-spindle intersection. Both dismantling the spindle and NE fusion depend on membrane-remodeling ESCRT-III filaments and enzymes such as the microtubule-severing enzyme,

55 SPASTIN, and the ESCRT-III disassemblase, VPS4 ${ }^{6,13,14}$. The crucial nature of deploying this pathway 
57 genomic instability and nuclear malformation ${ }^{6,13-16}$. There are twelve human ESCRT-III proteins that

58 polymerize on membrane surfaces to form membrane-remodeling filaments. Among these, CHMP7 is

59 unique because it contains N-terminal VPS25-like winged helix domains, followed by a C-terminal

60 ESCRT-III polymerization domain ${ }^{17}$. As part of the ESCRT-II complex, VPS25 acts to nucleate ESCRT-

61 III filament formation ${ }^{18,19}$. Interestingly, CHMP7 acts upstream of other ESCRT-IIIs that function at the

$62 \mathrm{NE}^{6,11,13}$. With CHMP7's non-canonical ESCRT-II/III hybrid architecture, it is not known whether

63 CHMP7 acts to polymerize other ESCRT-III proteins by its VPS25-like domains, whether it can

64 polymerize itself, or whether it integrates both features. While it is known that the LEM-domain protein

65 LEM2 interacts directly with CHMP7 and is required for its recruitment to the nuclear envelope ${ }^{11,12}$, it is

66 unclear how the ESCRT pathway is activated at specific sites where spindle disassembly must take place

67 in coordination with nuclear membrane remodeling. Our investigation of these questions has revealed a

68 new paradigm for nuclear envelope closure in which unexpected properties of LEM2, including the

69 ability to undergo phase separation and to co-polymerize with CHMP7, are key.

70 


\section{LEM2 forms liquid droplets for core localization.}

73 Using live cell imaging, we observed that full-length LEM2 transiently concentrated at the NE core and 74 colocalized with BAF as nuclear transport cargo began accumulating (Fig. 1A, Extended Data Fig. 1A-C,

75 Supplementary Table 1). The LEM domain of LEM2 bound BAF in vitro, and this interaction was necessary for LEM2 targeting (Fig. 1A, Extended Data Fig. 1C-E). Specifically, a four-amino acid (AA) substitution in the LEM domain (LEM $2_{\mathrm{m} 21}$ ), analogous to the $\mathrm{m} 24$ mutation described for the related

78 LEM family protein Emerin that precludes BAF binding ${ }^{20}$, disrupted LEM2 concentration at the core during this window.

Examination of LEM2's sequence reveals an extended low complexity domain (LCD, AA 40-213). LCDs can mediate phase separation via multivalent, low-affinity interactions that scaffold segregated, liquid biochemical environments ${ }^{21,22}$. Deletion of LEM2's LCD (LEM2 $\left.{ }_{\triangle 43-202}\right)$ disrupted LEM2 concentration at the core despite the presence of the LEM domain, indicating that the LEM domain and the LCD are each necessary but neither is sufficient for localization (Fig. 1A). LEM2's LCD diverges from the average protein's amino acid composition with increased Alanine (A) and Tryptophan (W) content, and two subdomains distinguished by elevated Serine (S) and Tyrosine (Y) or Arginine (R) and Proline (P) levels, respectively (Fig. 1A, Extended Data Fig. 1F-G). Other proteins with comparable compositional biases

88 form phase-separated liquid droplets ${ }^{22,23}$. To determine whether LEM2's LCD undergoes phase separation, we first analyzed the behavior of purified extra-lumenal domains of LEM2 in vitro. At physiological salt and $\mathrm{pH}$, we observed that the entire N-terminal domain, which includes the LEM motif and LCD (LEM2 $\left.2_{1-208}\right)$, spontaneously formed spherical droplets that displayed liquid-like properties: droplet fusion and inter- and intra-droplet diffusion (Fig.1B-D, Extended Data Movies 1 and 2). Interestingly, at higher concentrations, LEM2 $2_{1-208}$ droplets also spread out to wet the glass surface,

94 indicative of low surface tension (Fig.1B). Analyzing short, synthetic peptides tiled across LEM2's LCD 95 sequence revealed that the S-Y-rich region ${ }^{23}$ of LEM2's LCD was sufficient to form similar liquid 96 droplets that retained this wetting behavior on glass (Fig. 1E, Extended Data Fig. 2A). In summary, we 
97 found that the LEM and LCD domains of LEM2 are each required for LEM2 enrichment at the spindle-

98 containing core region of nascent nuclei through direct BAF binding and liquid-liquid phase separation,

99 respectively.

\section{A liquid coat for spindle microtubules.}

To better understand how LEM2's LCD mediates core enrichment, we performed stimulated emission depletion (STED) microscopy of immunostained HeLa cells. This imaging revealed that within the core region, endogenous LEM2 concentrates around spindle microtubules (MTs) near their interface with chromatin (Fig. 2A). In vitro analysis showed that the LEM $2_{1-208}$ droplets have the capacity to upconcentrate tubulin, and nucleated stabilized MT from soluble tubulin, at physiological salt and $\mathrm{pH}$ conditions that were otherwise prohibitive for tubulin polymerization in the absence of LEM $2_{1-208}$

108 (Extended Data Fig. 2B). Furthermore, LEM2 ${ }_{1-208}$ droplets bound to MTs and bundles of MTs, wetting the MT surface and coating them completely (Fig. 2B). As expected for a liquid coating, LEM2 ${ }_{1-208}$ found on MTs and MT bundles recovered its fluorescence after photobleaching, by contrast with the underlying MT lattice (Fig. 2B-C). (EM), revealed that the LEM2 LCD was required for the observed MT-bundling activity. Truncated protein constructs missing the LCD $\left(\mathrm{LEM}_{395-503}, \mathrm{LEM} 2_{1-72}\right)$ did not promote the formation of lightscattering MT bundles in vitro. Furthermore, MT bundle formation by LEM2 $2_{1-208}$ was concentration-

116 dependent and saturable, with a half-maximal scattering concentration of $1.3 \mu \mathrm{M}$ LEM2 ${ }_{1-208}$ (Fig. 2D,

117 Extended Data Fig. 2C). Negatively stained MT bundles detected by EM lost their characteristic tubulin 118 surface fine structure, occluded by the LEM $2_{1-208}$ coating (Fig. 2D-E). Using synthetic peptides that span

119 LEM2's LCD, we identified two separate sub-motifs within the P-R-rich region that were sufficient to 120 induce MT bundling (Fig. 2E, Extended Data Fig. 2A, D-G). Importantly, this P-R-rich region was also 
required for LEM2 core localization in living cells (Fig. 1A). In summary, the LCD's propensity for

122 phase separation and its affinity for microtubules govern LEM2's localization to spindle microtubules

123 during NE reformation, with the LEM domain further restricting this to the surface of chromatin disks.

\section{LEM2 and CHMP7 initiate sealing at the reforming NE.}

126

127

We previously reported that the C-terminal winged helix (WH) domain of LEM2, also referred to as the MSC domain ${ }^{11,24}$, directly binds to CHMP7 in vitro ${ }^{11}$. Testing the functional consequence of this binding interaction in vivo, we found that expression of a construct missing a portion of the WH-domain

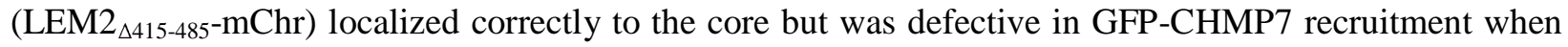
compared to the expression of full-length LEM2-mChr (Fig. 1A and 3A). This phenotype was dominant negative, as it was observed despite the presence of endogenous LEM2. Furthermore, overexpression of siRNA resistant $\mathrm{LEM} 2_{\triangle 415-485}-\mathrm{mChr}$ following depletion of endogenous LEM2 failed to rescue recruitment of IST1, an ESCRT-III protein known to be downstream of LEM2 and CHMP7 (Fig. 3B and C) ${ }^{6,11}$. In contrast, overexpression of siRNA resistant full-length LEM2 notably enhanced recruitment of endogenous IST1 to the nascent nuclear envelope, reflected by both elevated IST1 levels and premature recruitment (Fig. 3C, Extended Data Fig. 3A-C, Supplementary Table 1). Together, these observations indicate that the C-terminal WH-domain of LEM2 is necessary and sufficient to recruit CHMP7 and downstream ESCRT proteins to reforming nuclear membranes.

To test the biological role of LEM2's WH-domain in establishing nuclear compartmentalization, we measured initial stages of NLS cargo nuclear accumulation. We found that NLS-3xGFP began to accumulate prior to cleavage furrow ingression when full-length LEM2-mChr was overexpressed (Fig. 3D, Extended Data Fig. 3D). In contrast, the initiation of NLS-cargo nuclear accumulation was abrogated by expression of LEM $2_{\triangle 415-485}-\mathrm{mChr}$, indicating that LEM2 works directly with CHMP7 to promote nuclear compartmentalization during early anaphase (Fig. 3D, Extended Data Fig. 3D). 


\section{LEM2 activates CHMP7 to form looping copolymers.}

146 To elucidate how LEM2 engages CHMP7 for early nuclear compartmentalization and ESCRT-III

147 recruitment, we turned to in vitro approaches. Full-length human CHMP7 purified as a stable monomer, and incubation of CHMP7 with LEM2 ${ }_{1-208}$ had no discernable effect on CHMP7's oligomeric state. Incubation of CHMP7 with the C-terminal LEM2 ${ }_{395-503}$ WH domain, however, triggered the assembly of multi-layered, looping polymers with an average inner diameter of $\sim 50 \mathrm{~nm}$ (Fig. 3E). Liposomes were also sufficient to trigger polymerization of full-length CHMP7, with many polymers bound to the membrane surface (Extended Data Fig. 3E-F). 2D alignment and image averaging of these membraneinduced polymers, which were in a favorable orientation for subsequent classification, revealed a repeating polymeric unit comprised of a continuous polymeric strand studded with repeating perpendicular spikes (Fig. 3F, Extended Data Fig. 3G). The dimensions of the periodic features in this polymer match those of the homologous structure of "open" conformation human CHMP1B and yeast Snf7 polymers, suggestive of an ESCRT-III polymeric strand and protruding ESCRT-II-like tandem WHdomains $^{19,25,26}$ (Fig. 3F, Extended Data Fig. 3G, Extended Data Table 1). A truncated CHMP7 fragment (CHMP7 $229-453)$ comprised only of the ESCRT-III domain spontaneously polymerized into rings during purification, also with an average diameter of $\sim 50 \mathrm{~nm}$, but devoid of perpendicular spikes (Fig. 3G). The CHMP7 ESCRT-III domain-only polymers confirm the domain arrangement model of the full-length CHMP7 polymer, and are consistent with an autoinhibitory function for CHMP7's N-terminal WH domains. To probe the mechanism of LEM2-mediated activation of CHMP7 polymerization, we quantified CHMP7 polymerization with increasing concentrations of LEM22 $395-503$ and found that LEM2's

165 WH domain induced a stoichiometric concentration of CHMP7 to polymerize, consistent with activation 166 by co-polymerization rather than by nucleation (Fig. 3H). Together, these data show that CHMP7 can 167 polymerize into looping strands with a polymerizing ESCRT-III domain regulated, in part, by autoinhibitory VPS25-like domains. Interestingly, CHMP7 polymerization is both activated and stoichiometrically limited by LEM2 $2_{395-503}$. 


\section{A domain replacement mechanism controls CHMP7.}

171 To investigate the mechanism of CHMP7 autoinhibition and release by LEM2's WH-domain, we employed a quantitative isotopic labeling and cross-linking mass spectrometry (XL-MS) approach (Fig. 4A, Supplementary Table 2). Labeling monomeric CHMP7 with a heavy crosslinker and LEM2-induced polymers with a light crosslinker, we quantitatively compared crosslink enrichment between samples. We identified 24 cross-links specific to monomeric CHMP7. Hybrid peptide mapping revealed that the Nand C-termini of CHMP7 fold back to interact with each other and with the $\alpha 1-\alpha 3$ helices of CHMP7's ESCRT-III domain (Fig. 4A-B, and Extended Data 4A-E). These interactions were significantly reduced

CHMP7 molecules, and between adjacent LEM2 $2_{395-503}$ molecules, are consistent with the co-assembly of both proteins (Fig. 4A-B, Extended Data Fig. 4). Mutating conserved residues implicated by XL-MS, within CHMP7 $\alpha 1$-3, impaired LEM2 ${ }_{395-503}$-induced CHMP7 polymerization (Fig. 4C and D, Extended Data Fig. 4F-G). These data suggest that autoinhibited CHMP7 is activated by LEM2 $2_{395-503}$ binding to the a1-3 region of CHMP7, and this, in turn, enables copolymerization. In the copolymer conformation, CHMP7's N-terminal WH-domains are presumably displaced from interactions with the ESCRT-III domain and may thus become available for downstream ESCRT-III binding (Fig. 3F, Fig. 4D).

\section{NE reformation depends on the LEM2 pathway.}

To understand the biological consequences of LEM2 copolymerization with CHMP7 in nuclear envelope phenotype in interphase, and is lethal with extended time ${ }^{27}$. To address when nuclear defects arise, we 
195 synchronized cells following LEM2 depletion and imaged their progression from anaphase to late 196 telophase. While LEM2-depleted cells progressed to anaphase without noticeable defects, a strong nuclear 197 and tubulin-morphology phenotype began to emerge in late anaphase and persisted past telophase (Fig. 198 5A-C, Extended Data Fig. 5A-B, Movie 3). Depletion of LEM2 resulted in severely misshapen nuclei and 199 3D imaging revealed thick MT bundles piercing through the nucleus in a channel lined by nuclear 200 membrane (Fig. 5B, Extended Data Fig. 5). The significance of this nuclear morphology phenotype is 201 underscored by the appearance of DNA damage — a hallmark of defective nuclear integrity—in newly202 formed nuclei (Fig. 5D, Extended Data Fig. 5C).

203 Interestingly, depletion of endogenous LEM2 alongside expression of LEM2 ${ }_{\triangle 415-485}-\mathrm{mChr}$ proved more 204 deleterious to nuclear morphology at interphase than simply depleting LEM2 (Fig. 5E, Extended Data 205 Fig. 5D). Since the N-terminal region of LEM $2_{\triangle 415-485}-\mathrm{mChr}$ is intact, while the WH domain is not, this 206 observation highlights that focal enrichment at the spindle-NE-chromatin interface, a function of LEM2's 207 N-terminus, must be balanced by downstream ESCRT-III activity, a function of LEM2's C-terminus. 208 These activities were similarly integrated in vitro, where a construct bearing the extra-lumenal domains of

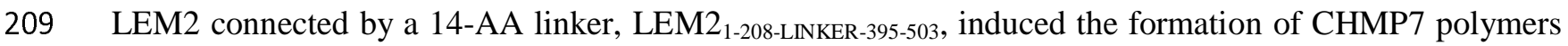
210 looping at and around small MT bundles (Fig. 5F). 
In this work we explain the molecular mechanisms underlying LEM2's function as a site-specific adaptor for mitotic ESCRT-III functions at the NE. Two distinct features specify the localization of LEM2 to sites along the nascent NE that are destined for coordinated membrane fusion and spindle disassembly: LEM domain interactions with BAF and its low complexity domain, which is responsible for liquid-liquid

217 phase separation and wetting the MT-chromatin interface. As an integral inner nuclear membrane protein,

218 LEM2 tethers the reforming NE to the chromatin surface and to the spindle, simultaneously recruiting the 219 ESCRT-II/ESCRT-III chimeric protein CHMP7 with its C-terminal WH-domain. We further reveal how 220 LEM2 initiates assembly of the ESCRT-III complex through direct activation of CHMP7. Together 221 LEM2 and CHMP7 copolymerize and loop around spindle MTs, creating a molecular "O-ring" to 222 promote nuclear compartmentalization, even before the spindle is fully disassembled (Extended Data Fig. 223 1A-B, Fig. 3D, Fig. 6).

224 Our data suggest that the polymeric surface of spindle MTs nucleates LEM2 condensation. We propose 225 that the deformable wetting behavior of LEM2 on the surface of MT bundles may serve as a fluid coating 226 at the junction where coalescing membranes encounter bundles of residual microtubules. As LEM2 is 227 embedded in the NE of cells, wetting of MT bundles in vivo may perform mechanical work by restricting 228 the size of gaps in the NE and drawing the membrane into close apposition with MT bundles before 229 ESCRT-III constriction and fusion of the membrane. We propose that during nuclear envelope 230 reformation LEM2 constitutes a deformable liquid phase between NE and spindle MT, surrounding 231 spindle MT bundles in a circular cross-section. This flexible toroid of LEM2 recruits and polymerizes 232 with CHMP7, in a LEM2-CHMP7 interaction that is required to initiate nuclear compartmentalization. 233 CHMP7, too, has a physical connection with the membrane at this junction ${ }^{28}$. Thus, we propose that the 234 LEM2-CHMP7 assembly provides a pliable circular connection between membrane and MT, pre-sealing 235 the NE prior to complete spindle disassembly and membrane fusion by the ESCRT-III pathway. These 236 results further raise the intriguing possibility that liquid-liquid phase separation of transmembrane or 
bioRxiv preprint doi: https://doi org/10.1101/577460; this version posted March 14, 2019. The copyright holder for this preprint (which was not certified by peer review) is the author/funder, who has granted bioRxiv a license to display the preprint in perpetuity. It is made available under aCC-BY-NC-ND 4.0 International license.

237 membrane-associated proteins provides important properties that may contribute to membrane remodeling 238 activities more generally ${ }^{29}$. 
241 Acknowledgments: We thank Wesley I. Sundquist and Sy Redding for critically reading the

242 manuscript. For reagents, technical advice, and discussions we thank the Frost and Ullman labs,

243 as well as the Nikon Imaging Center at UCSF. We thank Lauren Williams for helping score and

244 the lab of Michelle Mendoza for generously sharing microscope resources. We also thank the

245 UCSF Center for Advanced cryoEM, including Alexander Myasnikov, David Bulkley and

246 Michael Braunfeld.

247 Author contributions: Author contributions: A.v.A, D.L., I.E.J., M.T., A.L.B., K.S.U., and

248 A.F. designed research; A.v.A, D.L., I.E.J., M.T., and S.M.P. performed research; A.v.A, D.L.,

I.E.J., M.T., K.S.U., and A.F. analyzed data; and A.v.A, D.L., I.E.J., K.S.U., and A.F. wrote the paper.

Funding: Our research was supported by NIH grants P50 GM082545 and 1DP2-GM110772-

25201 (A.F.), the Huntsman Cancer Foundation and the Huntsman Cancer Institute Cancer Center

253 Support Grant NIH P30CA042014 (K.S.U.). Mass spectrometry analysis was co-funded by the

254 shared instrument grant for the mass spectrometer (NIH S10D016229) and the Dr. Miriam and

255 Sheldon G. Adelson Medical Research Foundation. A.v.A was funded by EMBO (ALTF 455-

256 2016) and the German Research Foundation (DFG AP 298/1-1). I.E.J. was funded by the NSF

257 Graduate Research Fellowship (1000232072) and a Mortiz-Heyman Discovery Fellowship.

258 Adam Frost is a Chan Zuckerberg Biohub investigator and an HHMI faculty scholar.

Competing interests: The authors declare no conflict of interest. 


\section{References:}

263 1. Wandke, C. \& Kutay, U. Enclosing chromatin: Reassembly of the nucleus after open mitosis. Cell (2013). doi:10.1016/j.cell.2013.02.046

2. Ungricht, R. \& Kutay, U. Mechanisms and functions of nuclear envelope remodelling. Nature Reviews Molecular Cell Biology 18, 229-245 (2017).

3. Kim, H.-S., Fernandes, G. \& Lee, C.-W. Protein Phosphatases Involved in Regulating Mitosis: Facts and Hypotheses. Mol. Cells 39, 654-662 (2016).

4. Otsuka, S. et al. Postmitotic nuclear pore assembly proceeds by radial dilation of small membrane openings. Nat. Struct. Mol. Biol. (2017). doi:10.1038/s41594-017-0001-9

5. Samwer, M. et al. DNA Cross-Bridging Shapes a Single Nucleus from a Set of Mitotic Chromosomes. Cell (2017). doi:10.1016/j.cell.2017.07.038

6. Vietri, M. et al. Spastin and ESCRT-III coordinate mitotic spindle disassembly and nuclear envelope sealing. Nature 522, 231-235 (2015).

275 7. LaJoie, D. \& Ullman, K. S. Coordinated events of nuclear assembly. Current Opinion in Cell Biology 46, (2017).

277 8. Haraguchi, T. et al. Live cell imaging and electron microscopy reveal dynamic processes of BAFdirected nuclear envelope assembly. J. Cell Sci. (2008). doi:10.1242/jcs.033597

279 9. Berk, J. M., Tifft, K. E. \& Wilson, K. L. The nuclear envelope LEM-domain protein emerin. Nucleus (United States) (2013). doi:10.4161/nucl.25751

10. Brachner, A. LEM2 is a novel MAN1-related inner nuclear membrane protein associated with Atype lamins. J. Cell Sci. (2005). doi:10.1242/jcs.02701 
yeast and human cells. Proc. Natl. Acad. Sci. 114, E2166-E2175 (2017).

285

286

287

288

289

290

291

292

293

294

295

296

297

298

299

300

301

302

303

304

305

12. Webster, B. M. et al. Chm7 and Heh1 collaborate to link nuclear pore complex quality control with nuclear envelope sealing. EMBO J. (2016). doi:10.15252/embj.201694574

13. Olmos, Y., Hodgson, L., Mantell, J., Verkade, P. \& Carlton, J. G. ESCRT-III controls nuclear envelope reformation. Nature 522, 236-239 (2015).

14. Ventimiglia, L. N. et al. CC2D1B Coordinates ESCRT-III Activity during the Mitotic Reformation of the Nuclear Envelope. Developmental Cell (2018). doi:10.1016/j.devcel.2018.11.012

15. Liu, S. et al. Nuclear envelope assembly defects link mitotic errors to chromothripsis. Nature (2018). doi:10.1038/s41586-018-0534-z

16. Vietri, M. et al. Unrestrained ESCRT-III drives chromosome fragmentation and micronuclear catastrophe. bioRxiv 517011 (2019). doi:10.1101/517011

17. McCullough, J., Frost, A. \& Sundquist, W. I. Structures, Functions, and Dynamics of ESCRTIII/Vps4 Membrane Remodeling and Fission Complexes. Annu. Rev. Cell Dev. Biol. (2018). doi:10.1146/annurev-cellbio-100616-060600

18. Im, Y. J. \& Hurley, J. H. Integrated Structural Model and Membrane Targeting Mechanism of the Human ESCRT-II Complex. Dev. Cell (2008). doi:10.1016/j.devcel.2008.04.004

19. Im, Y. J., Wollert, T., Boura, E. \& Hurley, J. H. Structure and Function of the ESCRT-II-III Interface in Multivesicular Body Biogenesis. Dev. Cell (2009). doi:10.1016/j.devcel.2009.07.008

20. Lee, K. K. et al. Distinct functional domains in emerin bind lamin A and DNA-bridging protein BAF. J Cell Sci (2001). doi:http://dx.doi.org/10.1016/B978-0-08-091812-9.50011-9

21. Banani, S. F., Lee, H. O., Hyman, A. A. \& Rosen, M. K. Biomolecular condensates: Organizers of 
307 22. Wang, J. et al. A Molecular Grammar Governing the Driving Forces for Phase Separation of Prion-like RNA Binding Proteins. Cell (2018). doi:10.1016/j.cell.2018.06.006

23. Hughes, M. P. et al. Atomic structures of low-complexity protein segments reveal kinked b sheets that assemble networks. Science (80-. ). (2018). doi:10.1126/science.aan6398

24. Caputo, S. et al. The carboxyl-terminal nucleoplasmic region of MAN1 exhibits a DNA binding winged helix domain. J. Biol. Chem. (2006). doi:10.1074/jbc.M601980200

25. McCullough, J. et al. Structure and membrane remodeling activity of ESCRT-III helical polymers. Science (80-. ). 350, 1548-1551 (2015). filaments. Elife (2015). doi:10.7554/eLife.12548

317 27. Ulbert, S., Antonin, W., Platani, M. \& Mattaj, I. W. The inner nuclear membrane protein Lem2 is critical for normal nuclear envelope morphology. FEBS Lett. (2006). doi:10.1016/j.febslet.2006.10.060 doi:10.1016/j.cub.2016.07.039

29. Milovanovic, D., Wu, Y., Bian, X. \& De Camilli, P. A liquid phase of synapsin and lipid vesicles. Science (80-. ). (2018). doi:10.1126/science.aat5671

325 30. Bradley, C. M., Ronning, D. R., Ghirlando, R., Craigie, R. \& Dyda, F. Structural basis for DNA bridging by barrier-to-autointegration factor. Nat. Struct. Mol. Biol. (2005). doi:10.1038/nsmb989

327 31. Hamon, L., Savarin, P., Curmi, P. A. \& Pastré, D. Rapid assembly and collective behavior of 
microtubule bundles in the presence of polyamines. Biophys. J. (2011).

doi:10.1016/j.bpj.2011.05.022

32. Mastronarde, D. N. Automated electron microscope tomography using robust prediction of specimen movements. J. Struct. Biol. (2005). doi:10.1016/j.jsb.2005.07.007

33. Bui, K. H. et al. Integrated structural analysis of the human nuclear pore complex scaffold. Cell 155, 1233-1243 (2013).

34. Leitner, A. et al. Expanding the Chemical Cross-Linking Toolbox by the Use of Multiple

Proteases and Enrichment by Size Exclusion Chromatography. Mol. Cell. Proteomics (2012).

35. Trnka, M. J., Baker, P. R., Robinson, P. J. J., Burlingame, A. L. \& Chalkley, R. J. Matching Cross-linked Peptide Spectra: Only as Good as the Worse Identification. Mol. Cell. Proteomics (2014). doi:10.1074/mcp.M113.034009

36. Schilling, B. et al. Platform-independent and Label-free Quantitation of Proteomic Data Using MS1 Extracted Ion Chromatograms in Skyline. Mol. Cell. Proteomics (2012).

37. Muller, F., Fischer, L., Chen, Z. A., Auchynnikava, T. \& Rappsilber, J. On the Reproducibility of Label-Free Quantitative Cross-Linking/Mass Spectrometry. J. Am. Soc. Mass Spectrom. 29, 405$412(2018)$.

38. Combe, C. W., Fischer, L. \& Rappsilber, J. xiNET: Cross-link Network Maps With Residue Resolution. Mol. Cell. Proteomics (2015). doi:10.1074/mcp.O114.042259

39. Kelley, L. A., Mezulis, S., Yates, C. M., Wass, M. N. \& Sternberg, M. J. E. The Phyre2 web portal for protein modeling, prediction and analysis. Nat. Protoc. 10, 845 (2015).

40. Pettersen, E. F. et al. UCSF Chimera - A visualization system for exploratory research and 
analysis. J. Comput. Chem. (2004). doi:10.1002/jcc.20084

352 41. Kosinski, J. et al. Xlink analyzer: Software for analysis and visualization of cross-linking data in

353 the context of three-dimensional structures. J. Struct. Biol. 189, 177-183 (2015).

354 42. Bajorek, M. et al. Biochemical Analyses of Human IST1 and Its Function in Cytokinesis. Mol. Biol. Cell (2009). doi:10.1091/mbc.E08-05-0475

43. Mackay, D. R., Makise, M. \& Ullman, K. S. Defects in nuclear pore assembly lead to activation of an Aurora B-mediated abscission checkpoint. J. Cell Biol. (2010). doi:10.1083/jcb.201007124

358 44. Mackay, D. R., Elgort, S. W. \& Ullman, K. S. The Nucleoporin Nup153 Has Separable Roles in Both Early Mitotic Progression and the Resolution of Mitosis. Mol. Biol. Cell (2009). doi:10.1091/mbc.e08-08-0883 
A

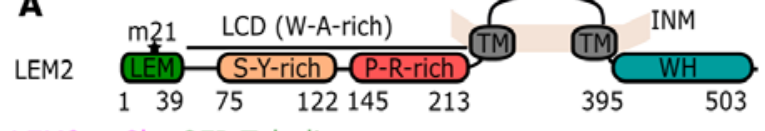

LEM2-mChr GFP-Tubulin

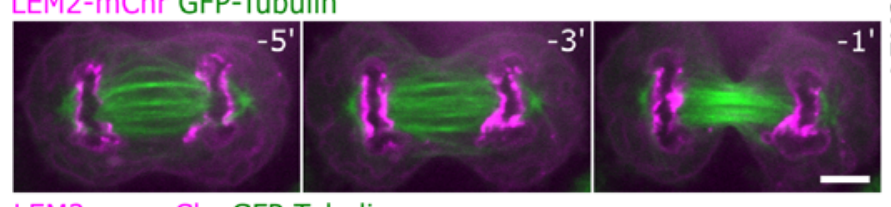

LEM2m21-mChr GFP-Tubulin

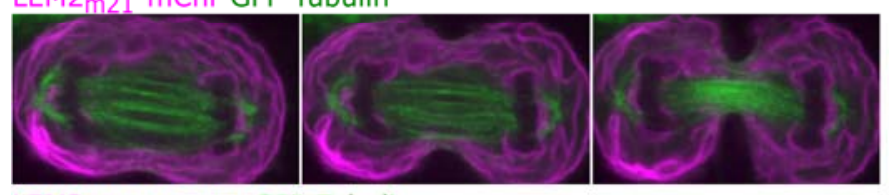

LEM2 $\triangle 43-202-m C h r G F P-T u b u l i n$

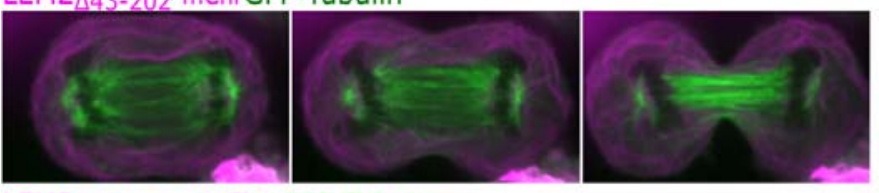

LEM2 $2_{\triangle 145-213-m C h r}$ GFP-Tubulin

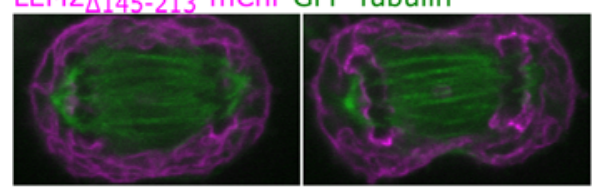

LEM2 ${ }_{\triangle 415-485-m C h r}$ GFP-Tubulin
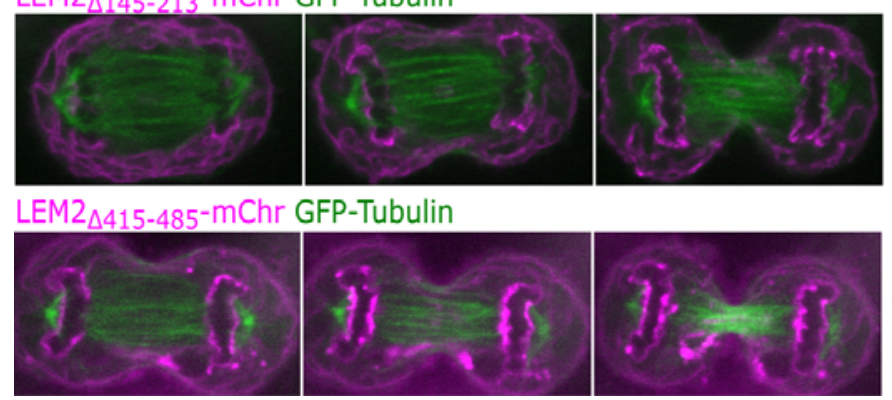
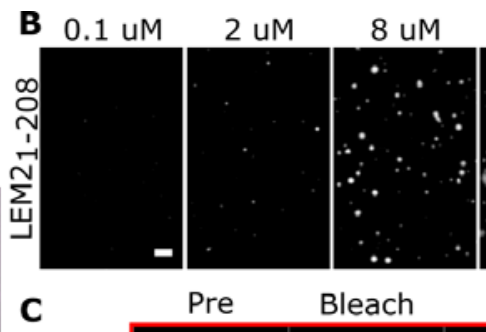

16 uM $32 \mathrm{uM}$
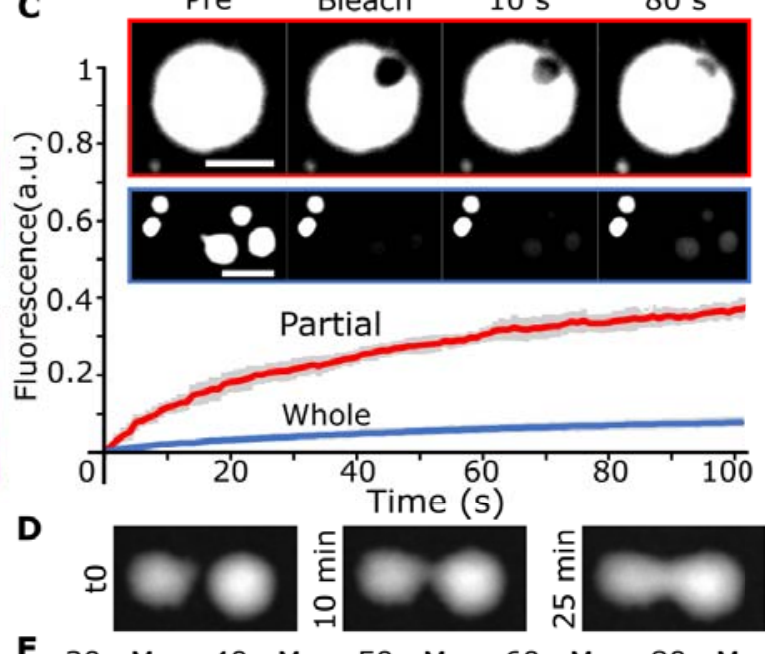

E 30 uM

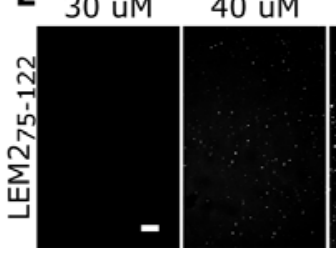

50 uM $60 \mathrm{uM}$

80 uM

Fig. 1. A low-complexity domain within LEM2 forms liquid droplets and is required for

core localization. (A) Top: domain architecture of LEM2. Bottom: Live cell localization of

LEM2-mChr and indicated deletions and mutations. Time 0 refers to full cleavage furrow

371 Real-time fluorescence imaging of LEM2 $2_{1-208}$-droplet fusion. (E) Concentration-dependent

372 droplet formation of S-Y rich peptide. Scale $5 \mu \mathrm{m}$ (A-E). 

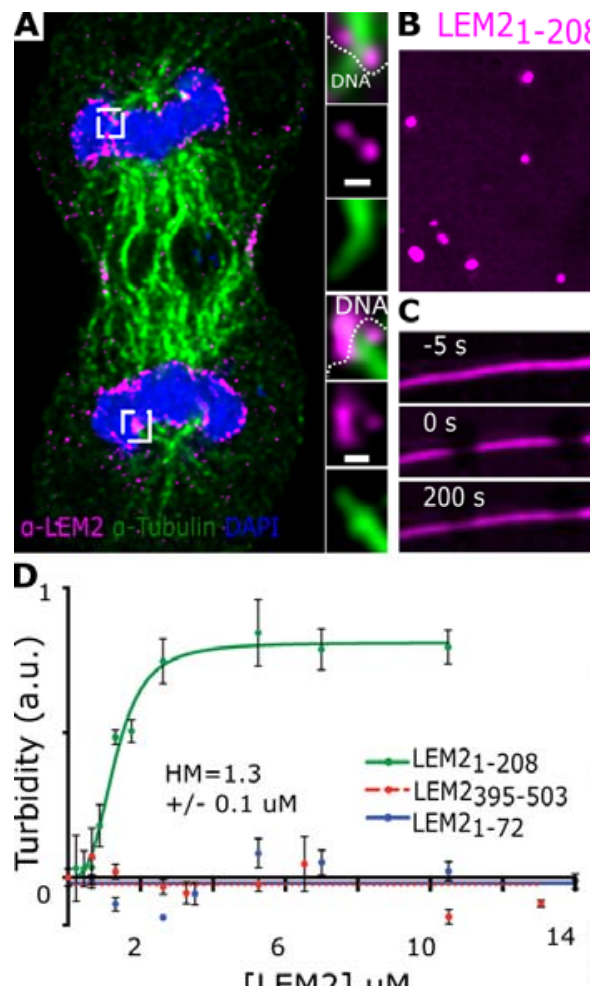

[LEM2] UM
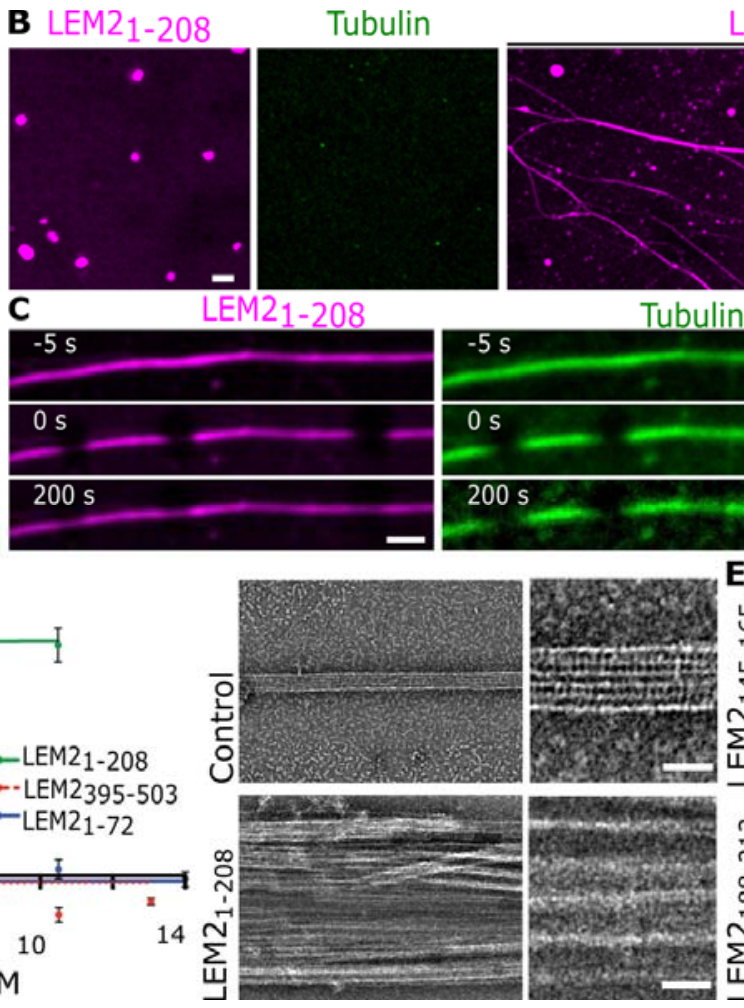

Tubulin
LEM21-208+Tubulin
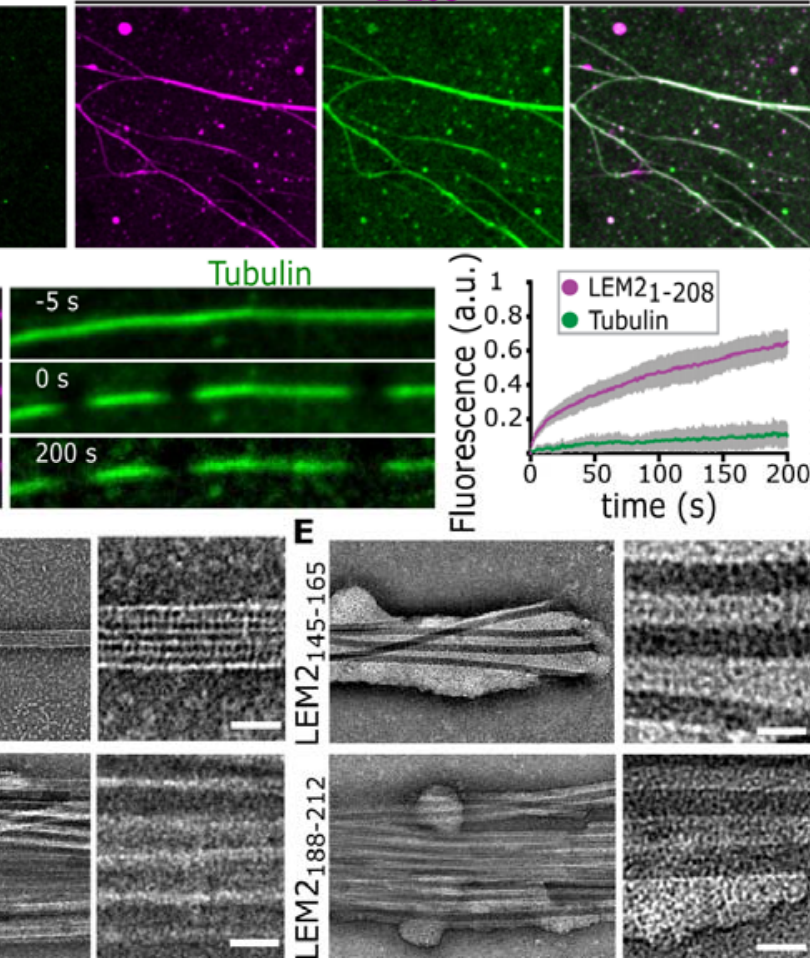

Fig. 2. LEM2's low complexity domain forms a liquid protein "coating" around spindle

378 bundles. $\mathrm{N}=17$, bars are SD, Scale $1 \mu \mathrm{m}$. (D) Light scattering-based quantification of MT bundling by

379 indicated constructs, bars are standard error of the mean (left) and electron micrographs showing LEM $2_{1-}$

380 208-coated MT bundles (right). Half Maximum (HM) (E) Electron micrographs showing MT bundles

381 induced by P-R-rich sequences from the LCD domain of LEM2. Scale $25 \mathrm{~nm}$ (D-E). 


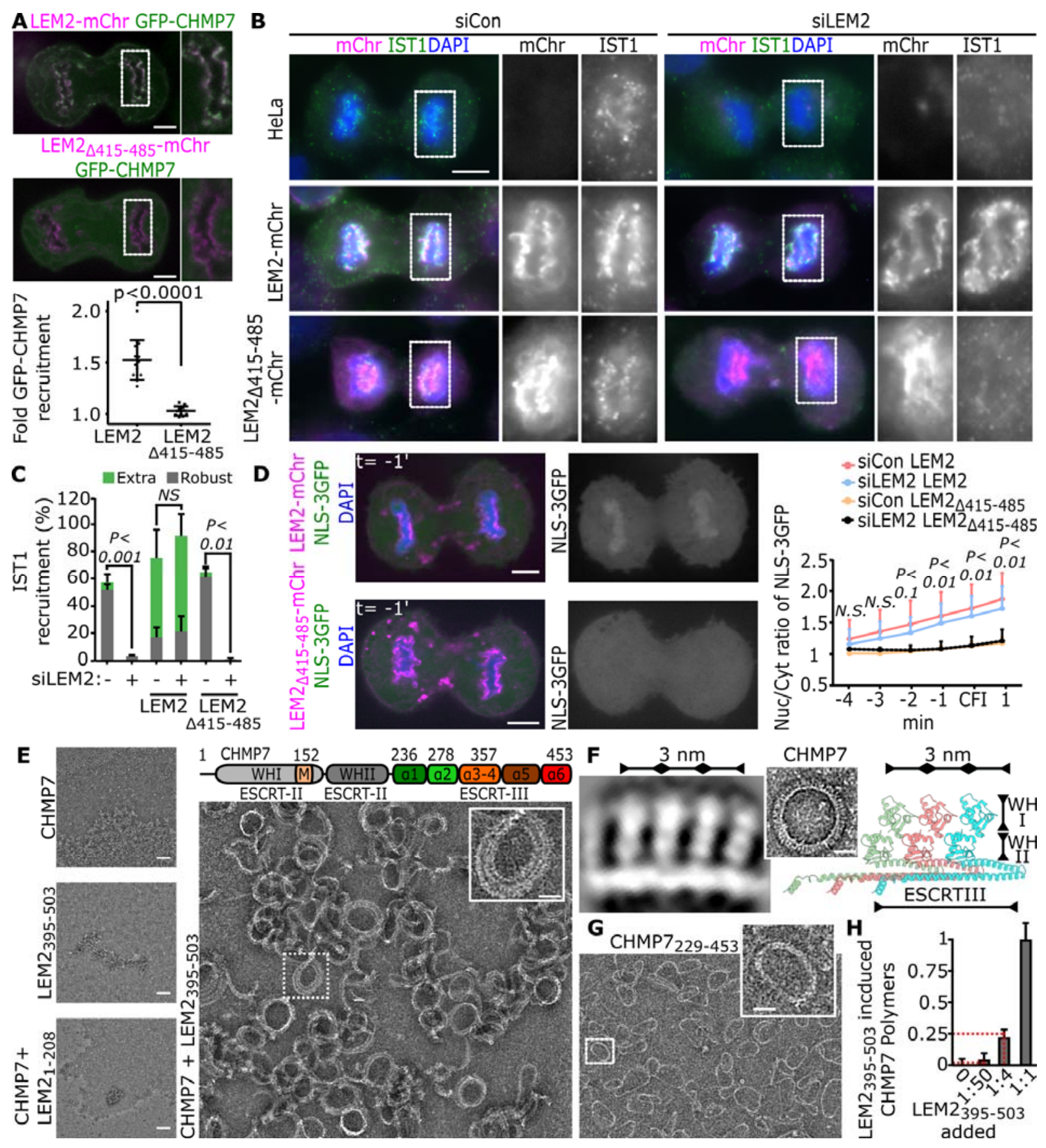

Fig. 3. LEM2's C-terminal WH domain induces CHMP7 polymerization and is required to seal the nascent NE. (A) Fold enrichment of GFP-CHMP7 at sites where full-length LEM2-mChr or LEM2 ${ }_{\triangle 415-}$ ${ }_{485}-\mathrm{mCh}$ concentrates at the NE in late anaphase. Bars are SD. (B) Recruitment of endogenous IST1 with

386 indicated siRNA treatments and expression of siRNA resistant constructs. (C) Quantification of robust

387 and extra-robust IST1 recruitment to chromatin disks in late anaphase. Error bars are standard error of the 
388 mean. Not significant (NS). (D) Nuclear import of NLS-3xGFP with indicated siRNA treatments and

389 expression of siRNA resistant constructs. Bars are SD. (E) CHMP7 polymerization assay with indicated

390 components assayed by negative stain EM. (F) Left: High magnification 2D class average of polymerized

391 CHMP7, Low-mag polymerized ring of full-length CHMP7 (inset). Right: Homology model of polymeric

392 CHMP7. (G) Negative stain EM of indicated construct. (H) SDS-page based relative quantification of

393 polymerized and pelleted CHMP7 with different ratios of LEM2 $2_{395-503}$ present. Red lines indicate

394 expected fraction of CHMP7 in the pelleted polymer, assuming 1:1 stoichiometric polymer. N=3. Error

395 bars are SD. Scale $10 \mu \mathrm{m}$ (A-D) $50 \mathrm{~nm}$ (E) $20 \mathrm{~nm}$ for insets (E-H). 
A

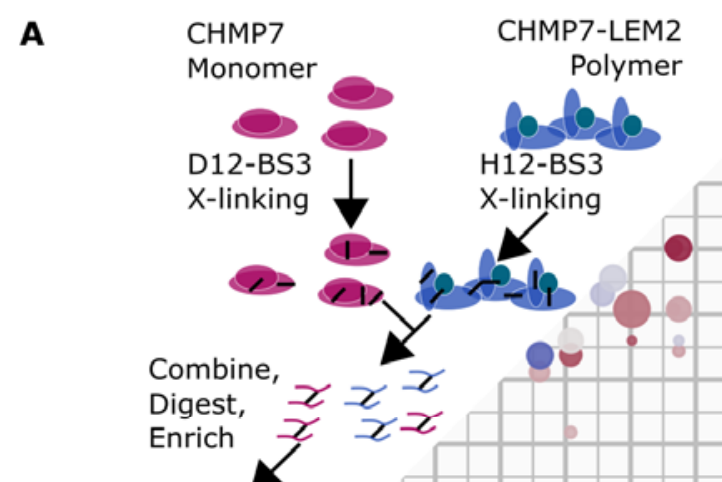

Mass Spectrometry based Identification and Quantification

Fig. 4. The WH domain of LEM2 activates CHMP7 polymerization by binding the ESCRT-III-core domain to relieve CHMP7 autoinhibition. (A) Workflow of lysine-lysine hybrid peptide mapping using XL-MS (top) and results represented by a domain interaction matrix (bottom). BS3 cross-links surface accessible Lys residues with $\mathrm{C} \alpha$-C $\alpha$ distances $<\sim 3 n m$. Each dot represents a pair of crosslinked Lys-Lys residues with area scaled to the sum of spectral counts and the color indicating the enrichment of each interaction in either monomeric CHMP7 (red) or LEM2 ${ }_{395-503}-\mathrm{CHMP7}$ polymer (blue). (B) Interactions 
404 that were enriched more than 4-fold are mapped on to the protein primary structure. Tested mutations are

405 indicated. MB, membrane binding. (C) Top: SDS-PAGE of protein in the pellet (P) or supernatant (S)

406 following centrifugation of LEM2 $2_{395-503}$ incubated with wild type or mutant CHMP7. Bottom: Recovery

407 in the pellet. Bars are SD of $n=3$. (D) Cartoon summary of LEM2-mediated CHMP7 activation. Arrows

408 illustrate the interaction of neighboring molecules identified by XL-MS. 

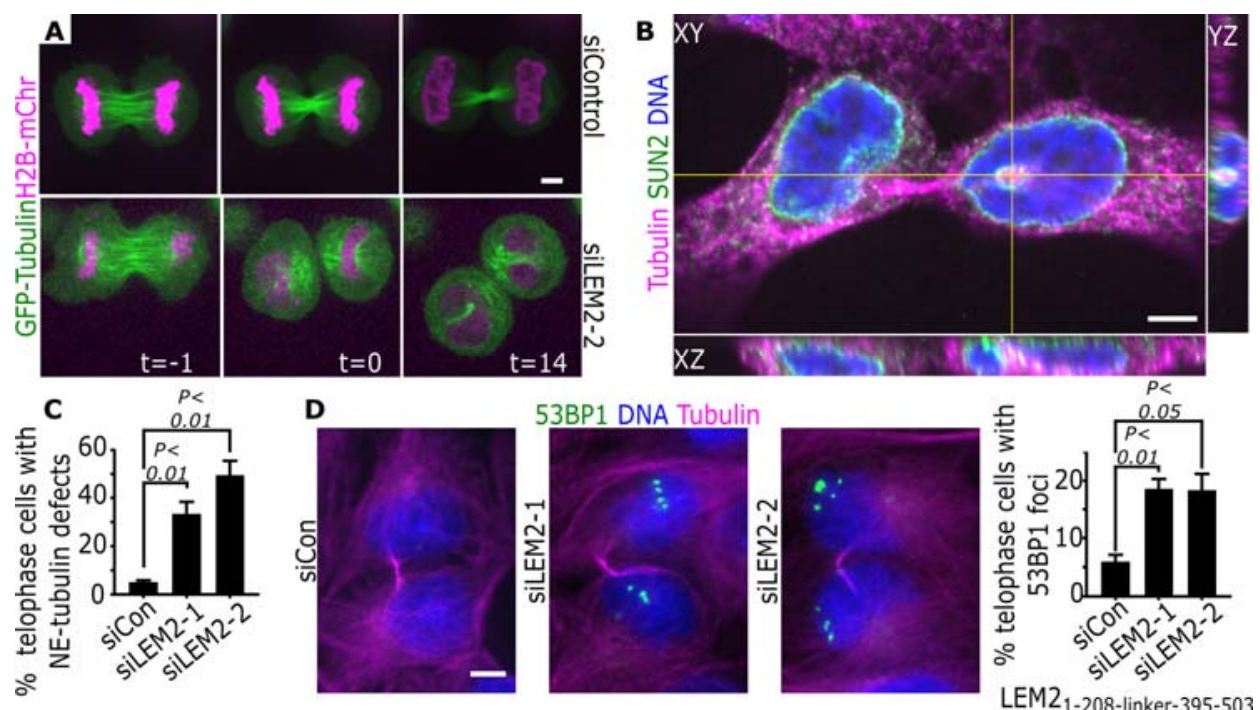

E

SiLEM2-2
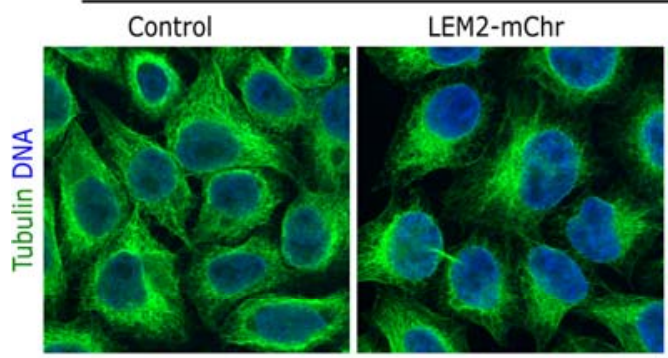

LEM2 ${ }_{\triangle 415-485}-\mathrm{mChr}$

$\mathbf{F}+\mathrm{CHMP7}+\mathrm{MT}$
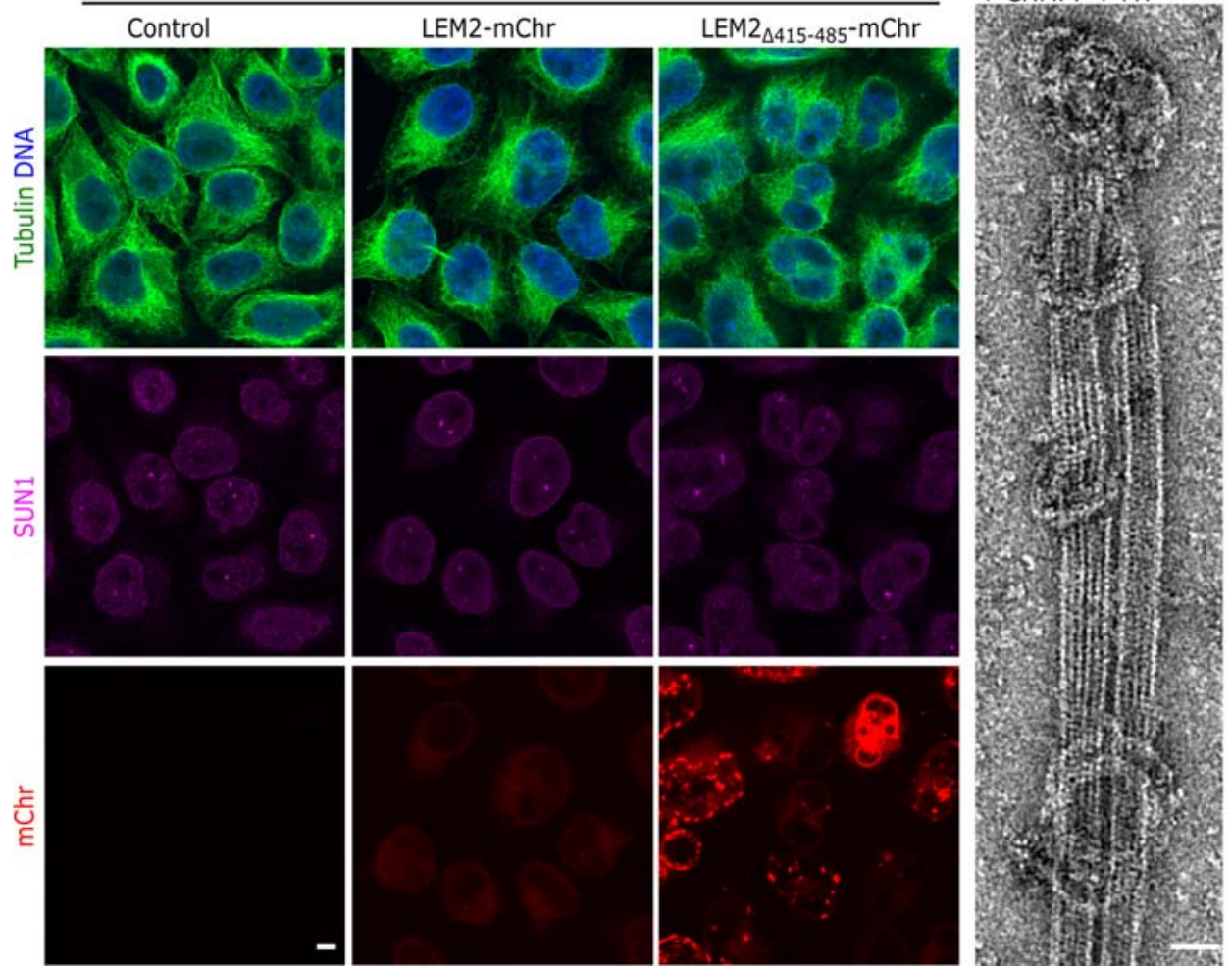

Fig. 5. LEM2 and ESCRT-III are essential for spindle disassembly and nuclear integrity.

412 (A) Live-cell imaging of GFP-Tubulin in siRNA treated cells. Time 0 refers to cleavage furrow

413 ingression in late anaphase. (B) Orthogonal view of a representative telophase cell following

414 treatment with siLEM2. (C) Tubulin defect scoring. Error bars are standard error of the mean.

415 (D) 53BP1 localization in telophase U2OS cells following siRNA treatment. Graph show percent 


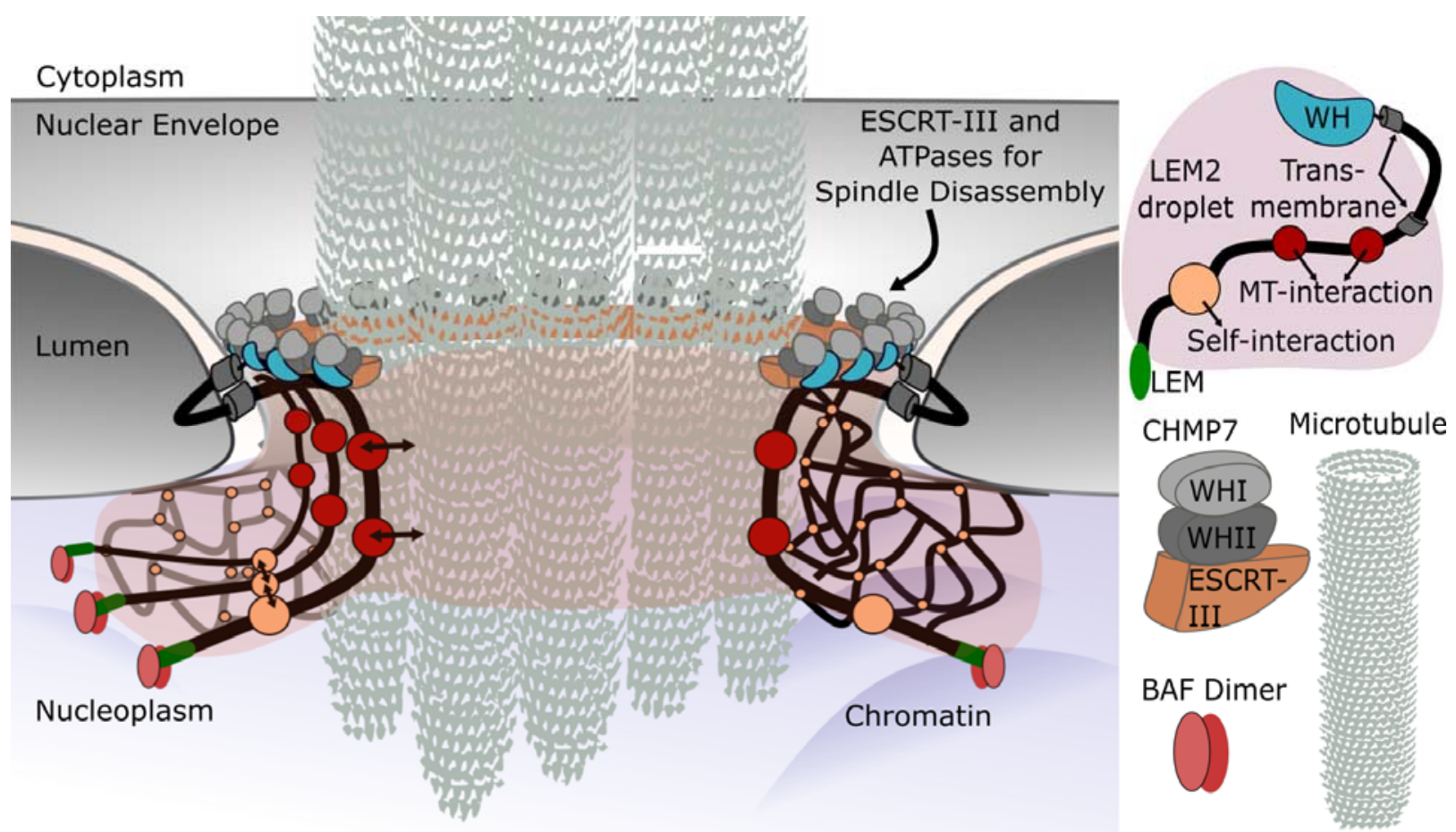

Fig. 6. Model of a molecular O-ring formed by LEM2 and CHMP7 during nuclear

423 deformable LEM2 phase forms around the spindle which is anchored to chromatin surface by

424 LEM2s interaction with BAF. LEM2 regulates ESCRT recruitment by inducing CHMP7

425 polymerization for nuclear membrane remodeling and sealing. 


\section{Extended Data information titles and legends}
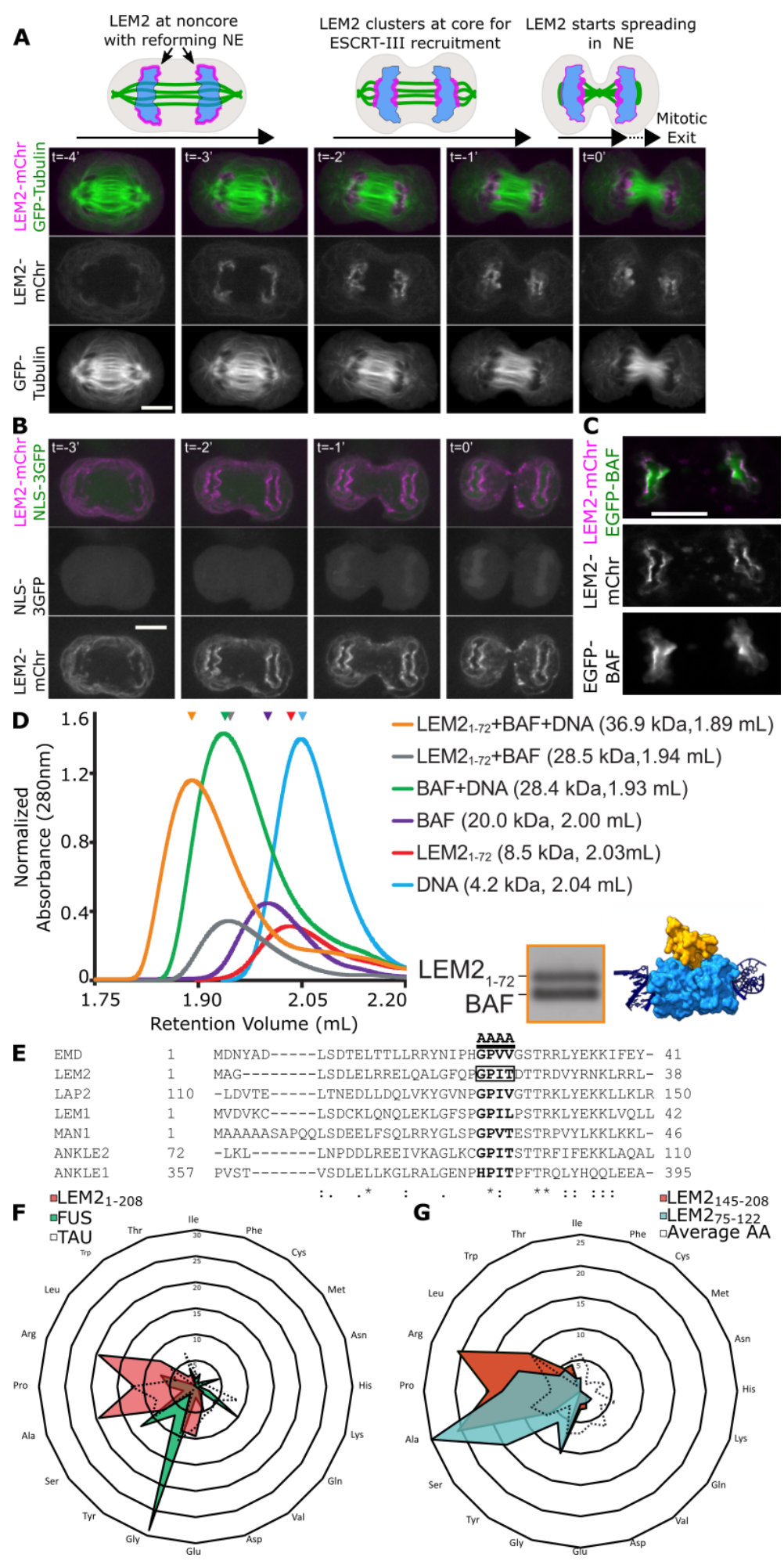

429 Extended Data Fig. 1. LEM2 dynamically colocalizes at the core with BAF. (A) Live

430 imaging of HeLa cells stably expressing LEM2-mCherry and GFP-Tubulin. The cartoon 
431 summarizes LEM2 localization through anaphase. Scale bar $10 \mu \mathrm{m}$. (B) HeLa cells stably

432 expressing NLS-3xGFP and transiently expressing LEM2-mCherry were live-imaged. Zero is

433 the time of complete cleavage furrow ingression (min). Scale bar $10 \mu \mathrm{m}$. (C) HeLa cells stably

434 expressing LEM2-mCherry and EGFP-BAF were live-imaged in anaphase. Scale bar $10 \mu \mathrm{m}$.(D)

435 Absorbance $280 \mathrm{~nm}$ as a function of retention volume (mL) from analytical size exclusion

436 chromatography. Retention volumes for major peaks (arrowheads) and predicted molecular

437 weights for protein or protein-DNA complexes are listed. Lower right: A homology model for

438 LEM2 ${ }_{1-72}$-BAF-DNA complex, based on high-resolution interfaces of BAF with DNA (2BZF),

439 and BAF with LEM-domain of EMERIN (2ODG) $(36,53)$. (E) Sequence alignment of LEM

440 domains across LEM family proteins showing conservation of a four-amino acid (AA) sequence

441 that, when mutated in EMD, disrupts BAF binding. Based on this alignment, the m21 mutation

442 in LEM2 is analogous to the m24 mutation in EMD (15). (F) LEM2 N-terminus (LEM2 $\left.{ }_{1-208}\right)$

443 percent amino acid composition compared to full length FUS and TAU proteins. (G) The LEM2

444 LCD shows elevated A and W levels compared to the average protein and has distinct S-Y-rich

445 (LEM2 $\left.{ }_{75-122}\right)$ and a R-P-rich (LEM2 $\left.{ }_{145-208}\right)$ subdomains. 
bioRxiv preprint doi: https://doi org/10.1101/577460; this version posted March 14, 2019. The copyright holder for this preprint (which was not certified by peer review) is the author/funder, who has granted bioRxiv a license to display the preprint in perpetuity. It is made available under aCC-BY-NC-ND 4.0 International license.

A LEM2 $40-60$ 40-GEARLRDEERLREEARPRGEE-60

LEM2 $21-87 \quad 61-$ RLREEARLREDAPLRARPAAASPRAEP-87

LEM2 $2_{75-122}$ 75-RARPAAASPRAEPWLSQPAS GSAYATPGAYGDIRP SAASWVGSRGLAY-122

LEM2123-144 123-PARPAQLRRRA SVRGSSEEDED-144

LEM2 $145-165$ 145-ARTPDRA TQGPG LA ARRWWAA-165

LEM2 $166-167$ 166-SPAPARLPS L L GPDPRPGLRA-187

LEM2 $188-212$ 188-TRA GPAGAARARPEVGRRLERWL SR-212
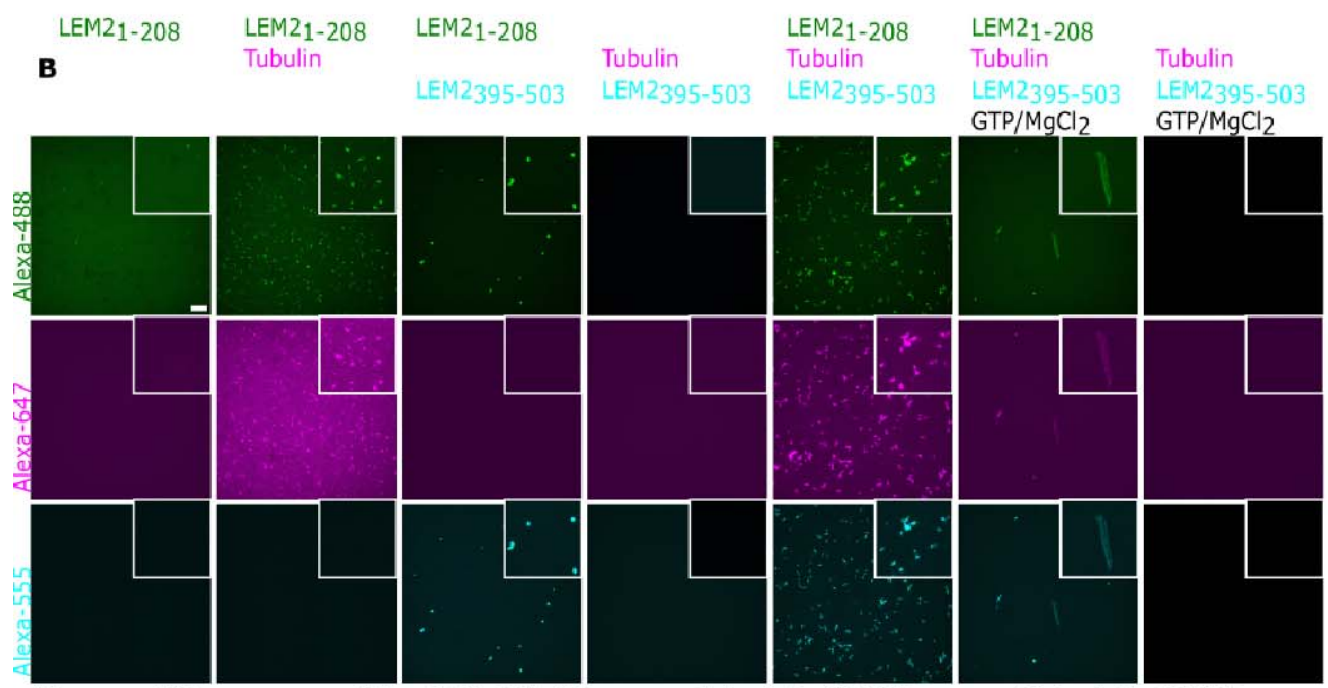

$\mathrm{GTP} / \mathrm{MgCl} 2$

$\mathrm{GTP} / \mathrm{MgCl} 2$
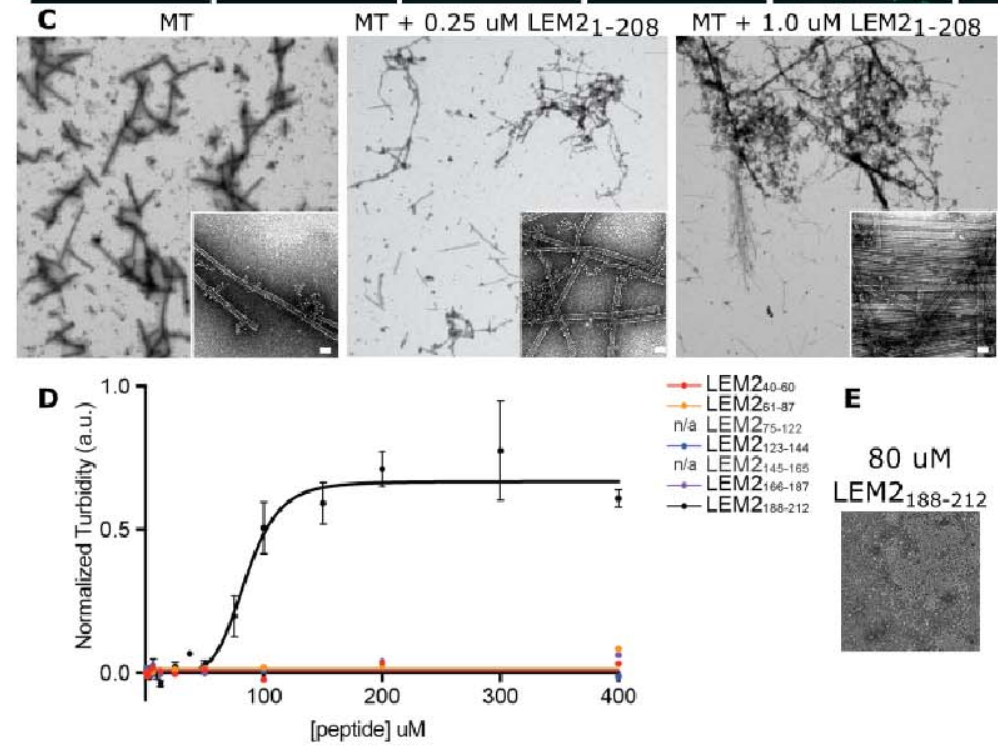

MT + 4.0 uM LEM $21-208$
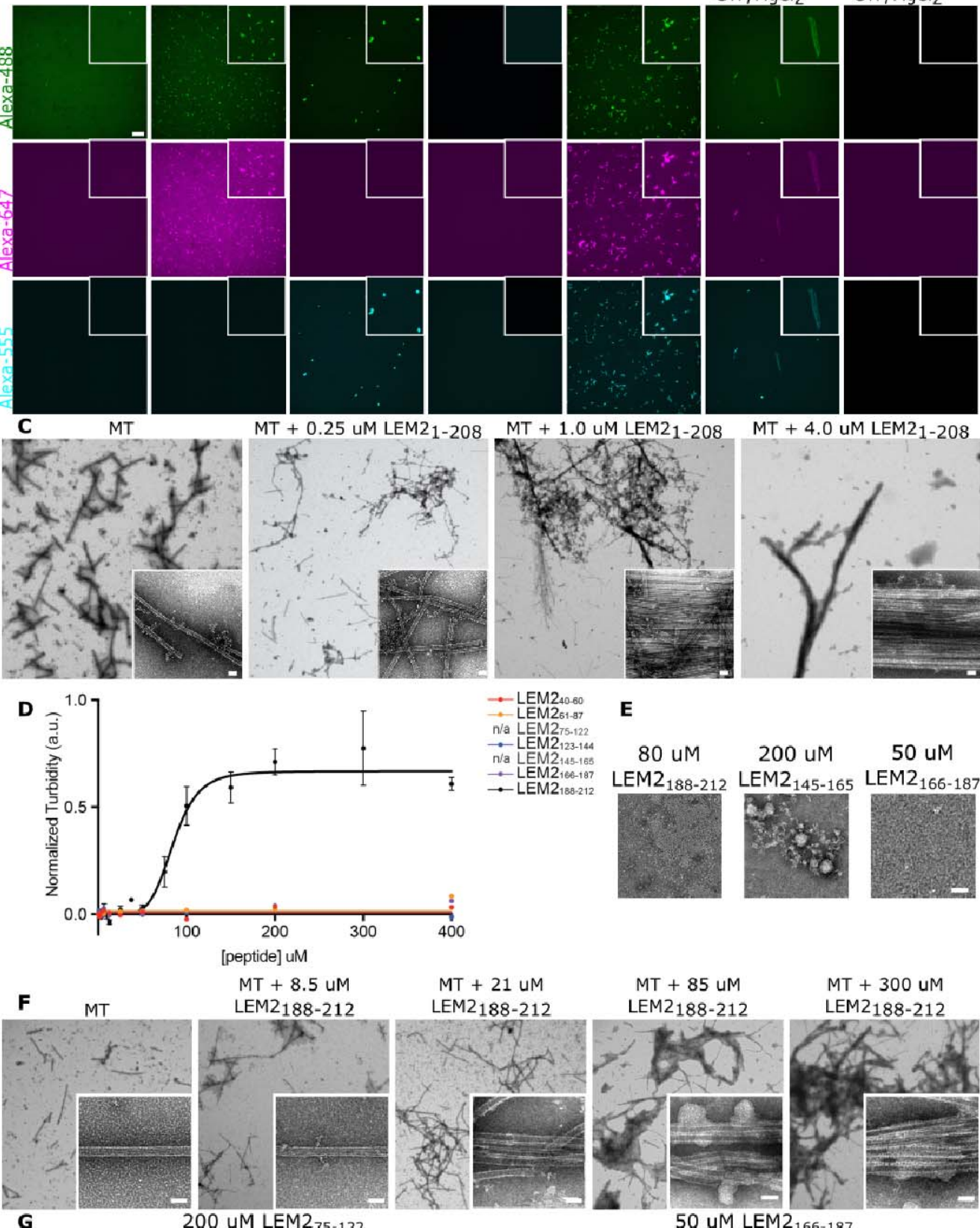

G

200 uM LEM2 $75 \cdot 12$

$\rightarrow$ LEM 240.60

$\rightarrow$ n/a LEM 2 $275-122$

$\mathbf{E}$

n/a LEM2 245-10 80 uM 200 uM 50 uM

$\rightarrow-L E M 2$
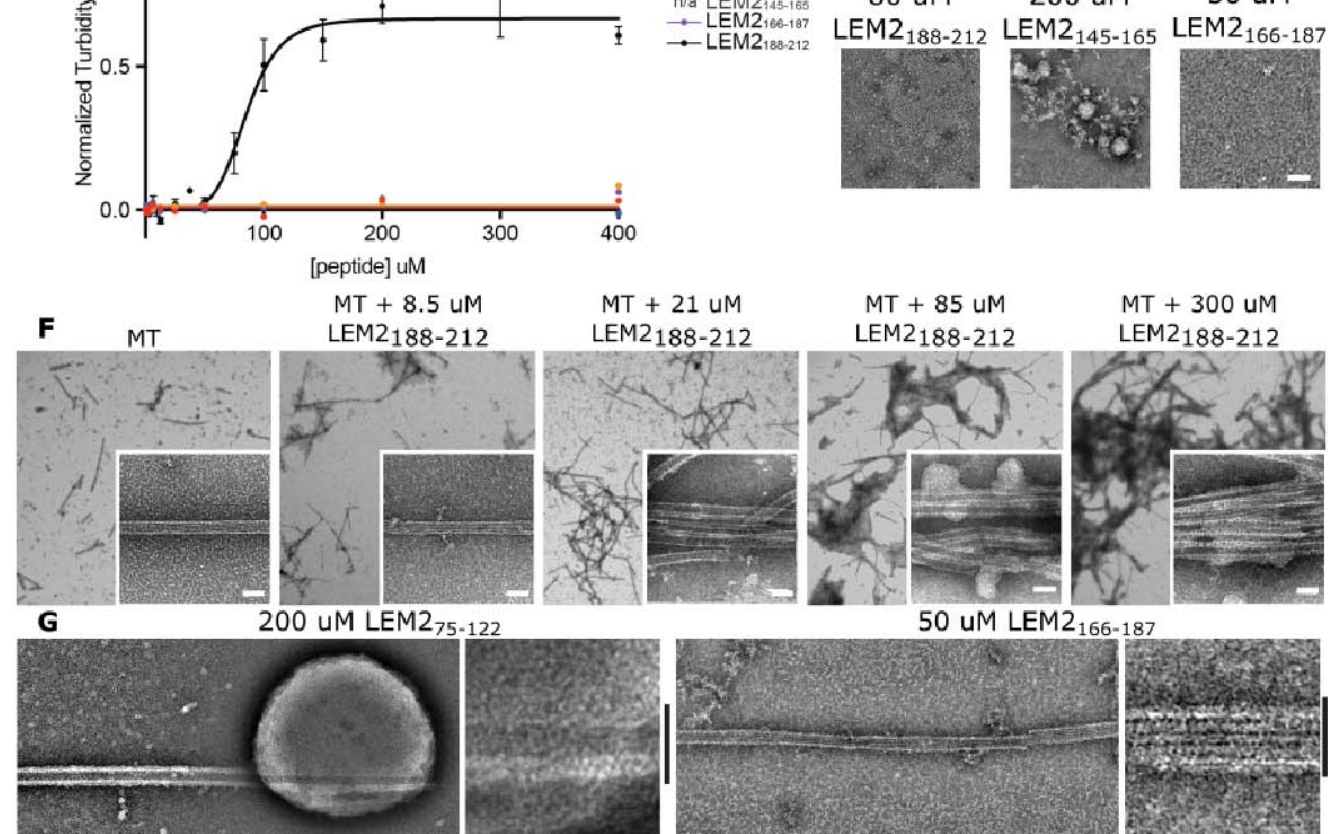
449 MT-bundling domains. (A) Amino acid sequences of 7 consecutive LEM2 peptides tiling the LCD. (B)

450 Indicated proteins, $8 \mu \mathrm{M}$ each, were incubated at physiological salt and pH. LEM2 was supplemented

451 with $0.5 \mu \mathrm{M}$ Alexa488-labeled His $_{6}$-SUMO-LEM2 ${ }_{1-208}$ for visualization. Scale bar is $10 \mu \mathrm{m}$. (C) Negative

452 stain EM of MTs or MTs with different concentrations of LEM2 $2_{1-208}$, corresponding to light scattering

453 reactions. Scale bar $25 \mathrm{~nm}$. (D) Light scattering-based quantification of MT bundling by indicated LEM2

454 peptides. Half maximal concentration of LEM2 $2_{188-212}$ is $85.11+/-3.490 \mu \mathrm{M}$. LEM $2_{75-122}$ and LEM $2_{145-165}$

455 peptides were turbid in the absence of MTs, and each induced the formation of clusters too big to be

456 analyzed by this assay upon exposure to MTs. (E) Representative negative stain EM of soluble LEM2

457 peptides (LEM2 $2_{166-187}$, LEM2 $2_{188-212}$ ), and a light scattering LEM2 peptide (LEM2 $2_{145-165)}$ ). Scale bar is 50

$458 \mathrm{~nm}$. (F) Negative stain EM of MTs or MTs with different concentrations of LEM2 $188-212$, corresponding to

459 light scattering reactions. Scale bar $25 \mathrm{~nm}$. (G) Negative stain EM of LEM2 $2_{75-122}$ or LEM2 $2_{166-187}$ with

460 MTs. Scale bars $25 \mathrm{~nm}$.

461 


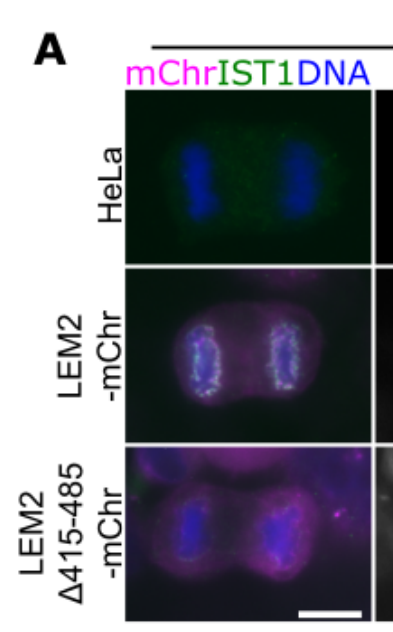

B

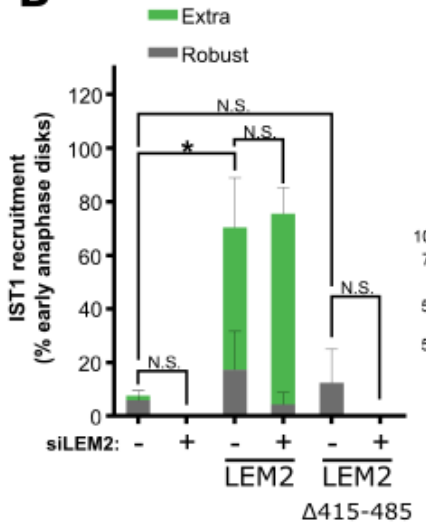

E

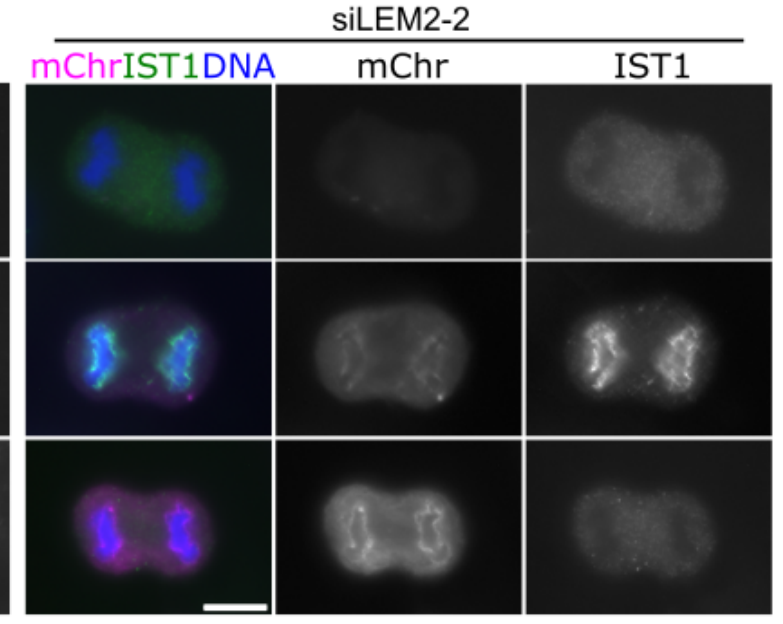

C
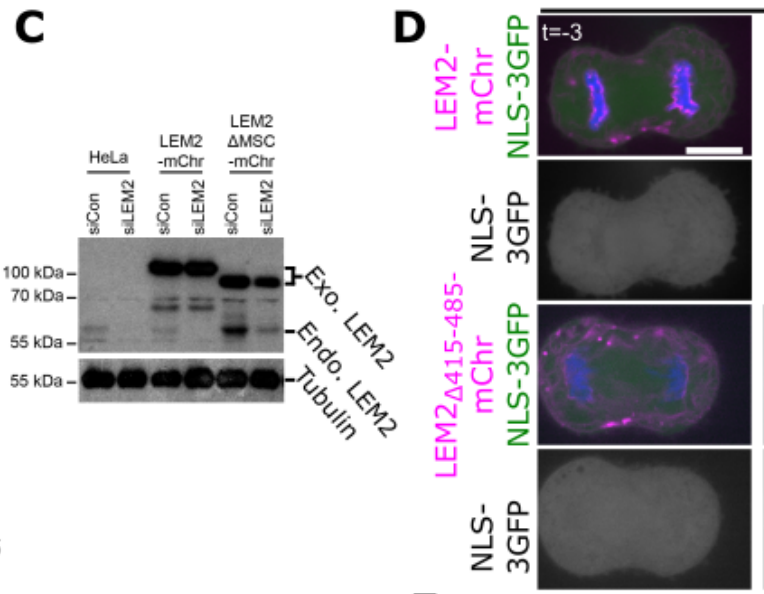

F

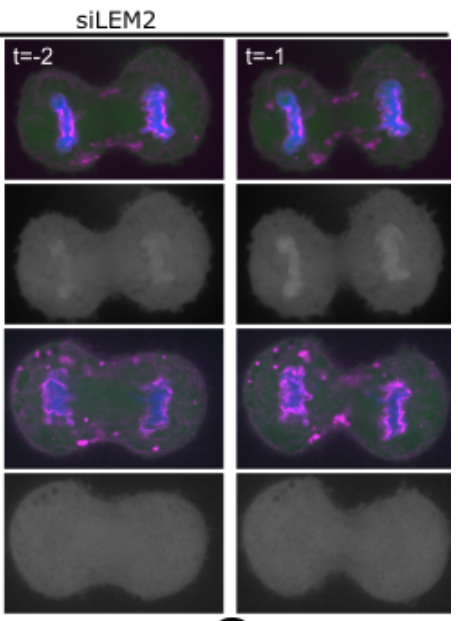

G
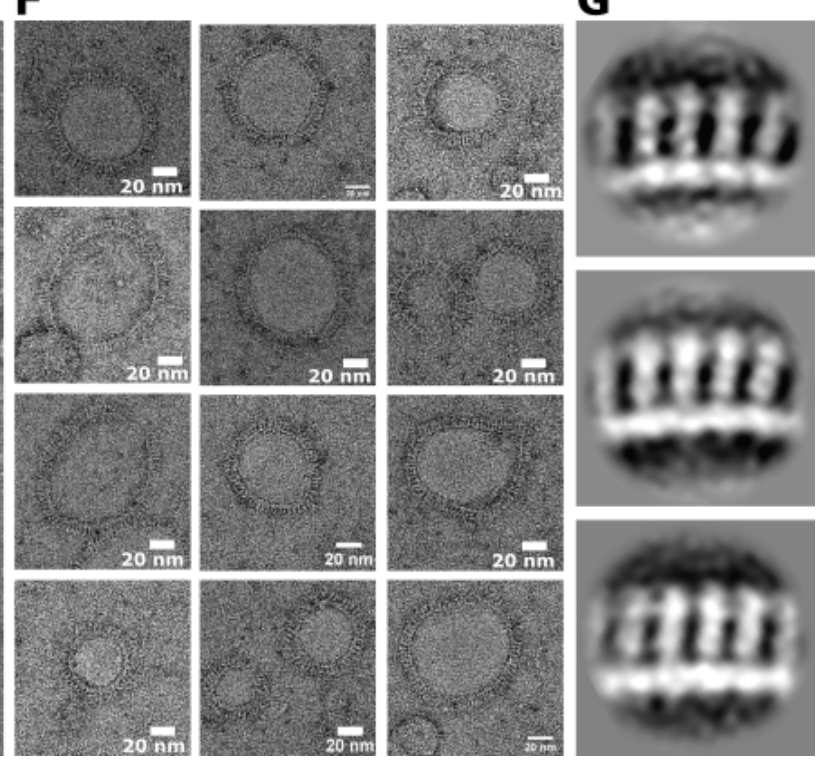

Extended Data Fig. 3. LEM2's C-terminal WH domain is required for IST1 recruitment

and nuclear compartmentalization. (A) Recruitment of endogenous IST1 with indicated 
465 siRNA treatment and expression of siRNA resistant constructs in early anaphase. Scale bars 10

466 um. (B) Quantification of the percent of early anaphase disks with robust and extra robust IST1

467 recruitment, as assessed by blind scoring. Bars represent the average of three independent

468 experiments and are graphed showing standard error of the mean. Student's t-test was used to

469 determine p-values, which are indicated as follows: $* P<0.05$; N.S., not significant. (C)

470 Immunoblot showing overexpression of the siRNA-resistant full length LEM2-mCherry (84kDa)

471 and LEM2 $2_{\Delta 415-485-\mathrm{mChr}}(76 \mathrm{kDa})$. Endogenous LEM2 is approximately 60kDa. (D)

472 Representative examples of HeLa cells treated with siLEM2 and expressing the indicated

473 siRNA-resistant LEM2 constructs and a nuclear localization signal fused to three GFPs (NLS-

$4743 \times$ GFP), live-imaged through anaphase. Scale $10 \mathrm{um}$. (E) Negative stain EM of CHMP7

475 polymers covering a liposome. Scale bar $100 \mathrm{~nm}$. (F) Negative stain EM of membrane-induced

476 CHMP7 polymers used for class-averaging. Scale bar $20 \mathrm{~nm}$. (G) Representative 2D-class

477 averages from manually particles from polymers shown in $\mathrm{F}$. 
A

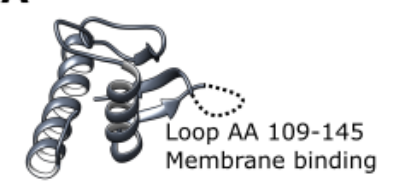

CHMP7 WH1

(AA 42-153)

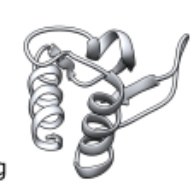

CHMP7 WH2

(AA 155-223)

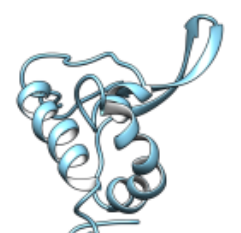

(y) LEM2-WH (AA 398-501)
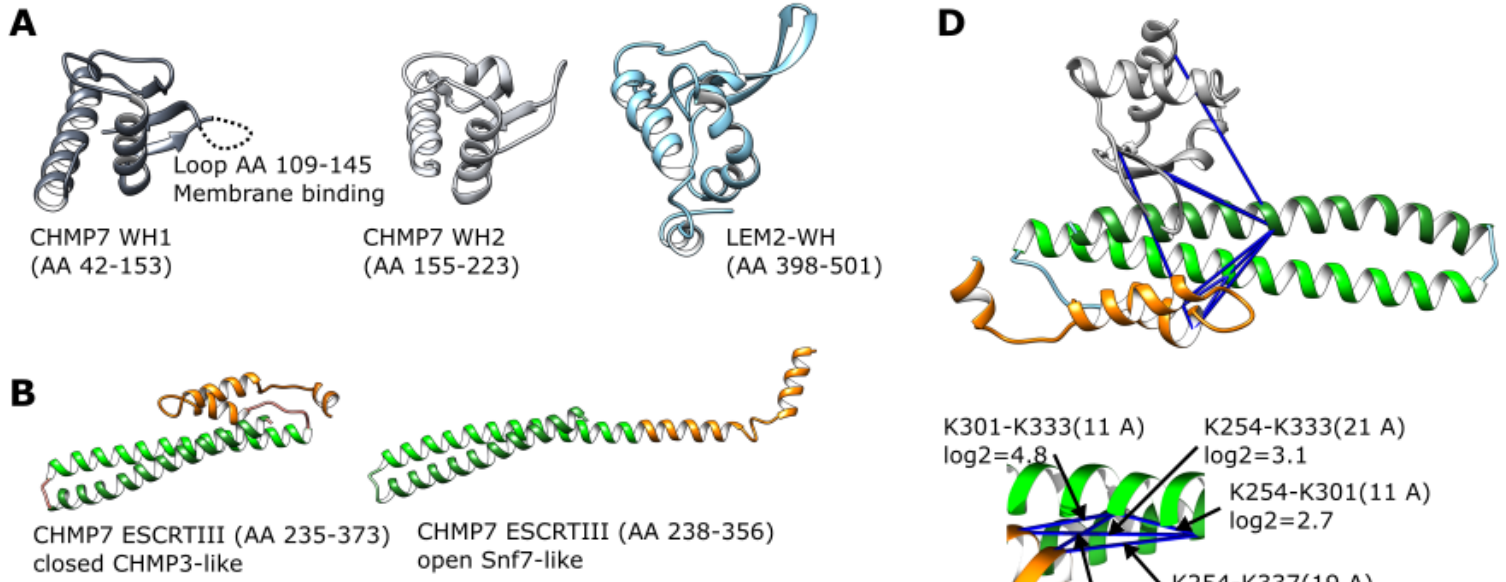

C

$\begin{array}{ll}\text { CHMP7 ESCRTIII (AA 235-373) } & \text { CHMP7 ESCRTI } \\ \text { closed CHMP3-like } & \text { open Snf7-like }\end{array}$

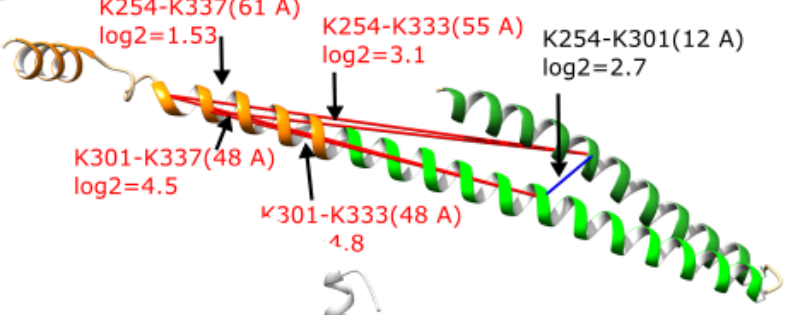

$\mathbf{E}$
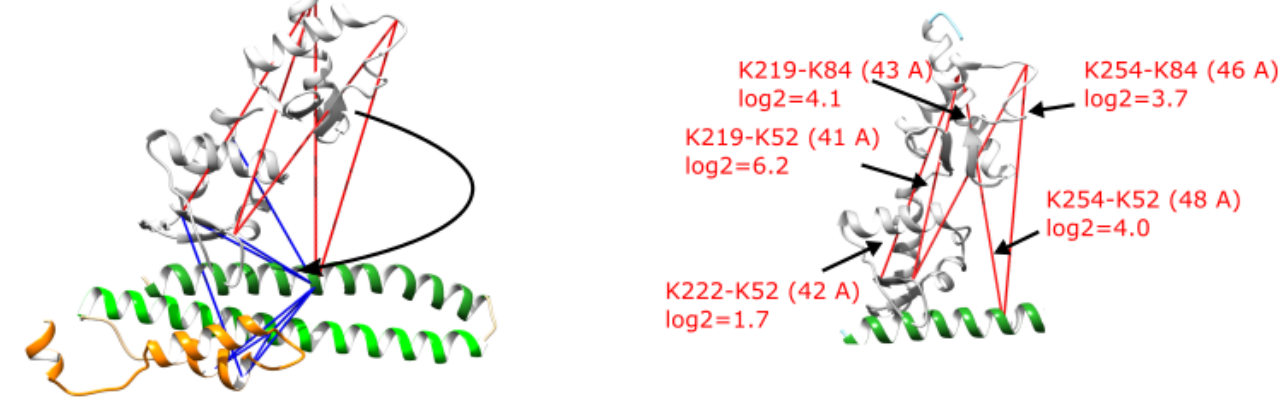

$\mathbf{F}$

G $\quad \operatorname{CHMP7}(\mathrm{V} 339 \mathrm{D}+\mathrm{I} 312 \mathrm{~W})$

+ LEM2595-503
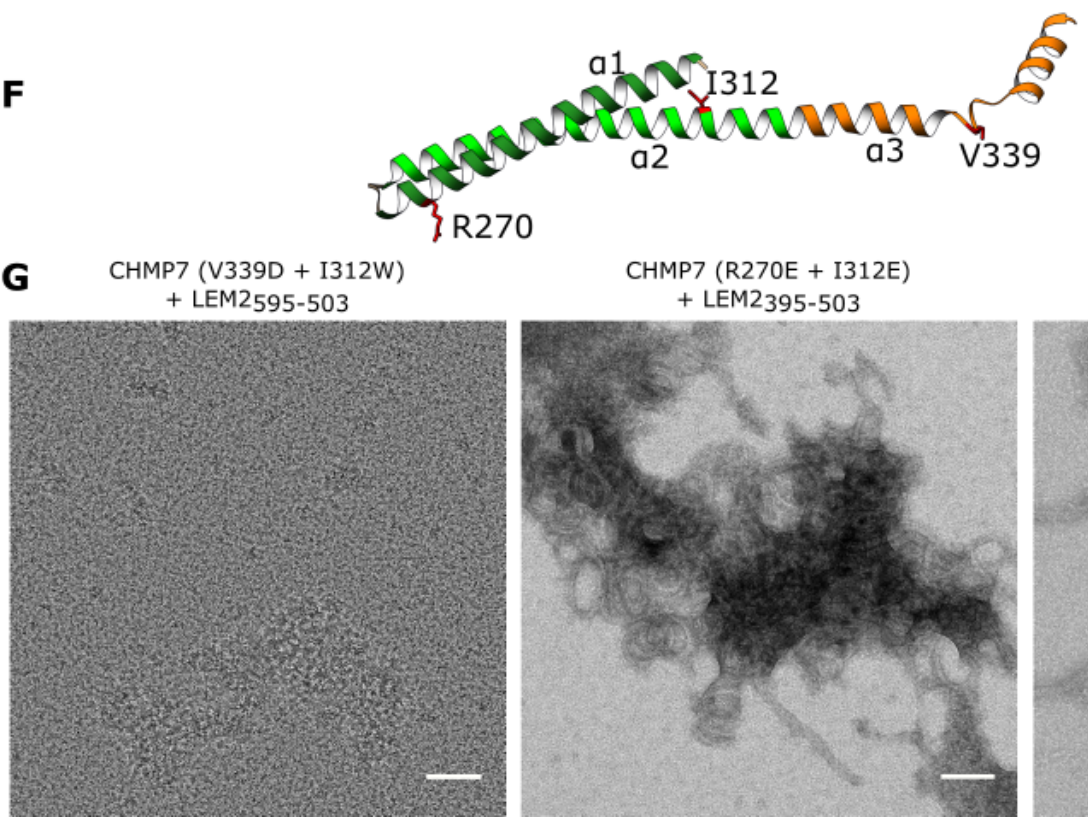

CHMP7 (R270E + I312E)

+ LEM2395-503

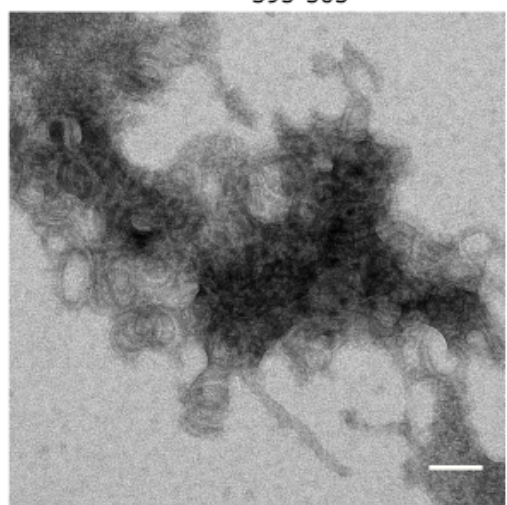

CHMP7 (V339D + R270E) +LEM2395-503 

lysine residues are indicated together with $\log _{2}$ fold change as compared to the polymeric CHMP7-LEM2 ${ }_{395-503}$ sample. Mapped $\mathrm{C} \alpha-\mathrm{C} \alpha$ distances $>3 \mathrm{~nm}$ are considered violations. (D) A

WH2 interaction to the CHMP7 ESCRTIII domain. All cross-links are satisfied when mapped to the closed CHMP7-ESCRTIII model (middle and lower panel) and agree with domain 492 connectivity. A subset of cross-links was not satisfied when mapping WH1 instead to same interface (data not shown). (E) The cross-linking data from monomeric CHMP7 suggests a hinge region between CHMP7 WH1 and WH2 that allows its WH1 to move closer to the ESCRTIII 495 core as compared to the conformation of polymerized CHMP7 in the class averages. (F) CHMP7 496 point mutations are shown in an open ESCRTIII-fold, representing polymerized CHMP7. (G) 497 CHMP7 mutants were dialyzed with His ${ }_{6}-$ SUMO-LEM2 ${ }_{395-503}$ in a 1:2 molar ratio, and any 498 resulting polymer was enriched by centrifugation and analyzed by negative stain EM. $499 \mathrm{CHMP}_{\mathrm{V} 339}+\mathrm{I}_{312 \mathrm{E}}$ did not form polymers in the presence of His 6 -SUMO-LEM2 ${ }_{395-503}$ during 500 dialysis. Scale bars are $50 \mathrm{~nm}$. 
bioRxiv preprint doi: https://doi.org/10.1101/577460; this version posted March 14,2019 . The copyright holder for this preprint (which was not certified by peer review) is the author/funder, who has granted bioRxiv a license to display the preprint in perpetuity. It is made available under aCC-BY-NC-ND 4.0 International license.

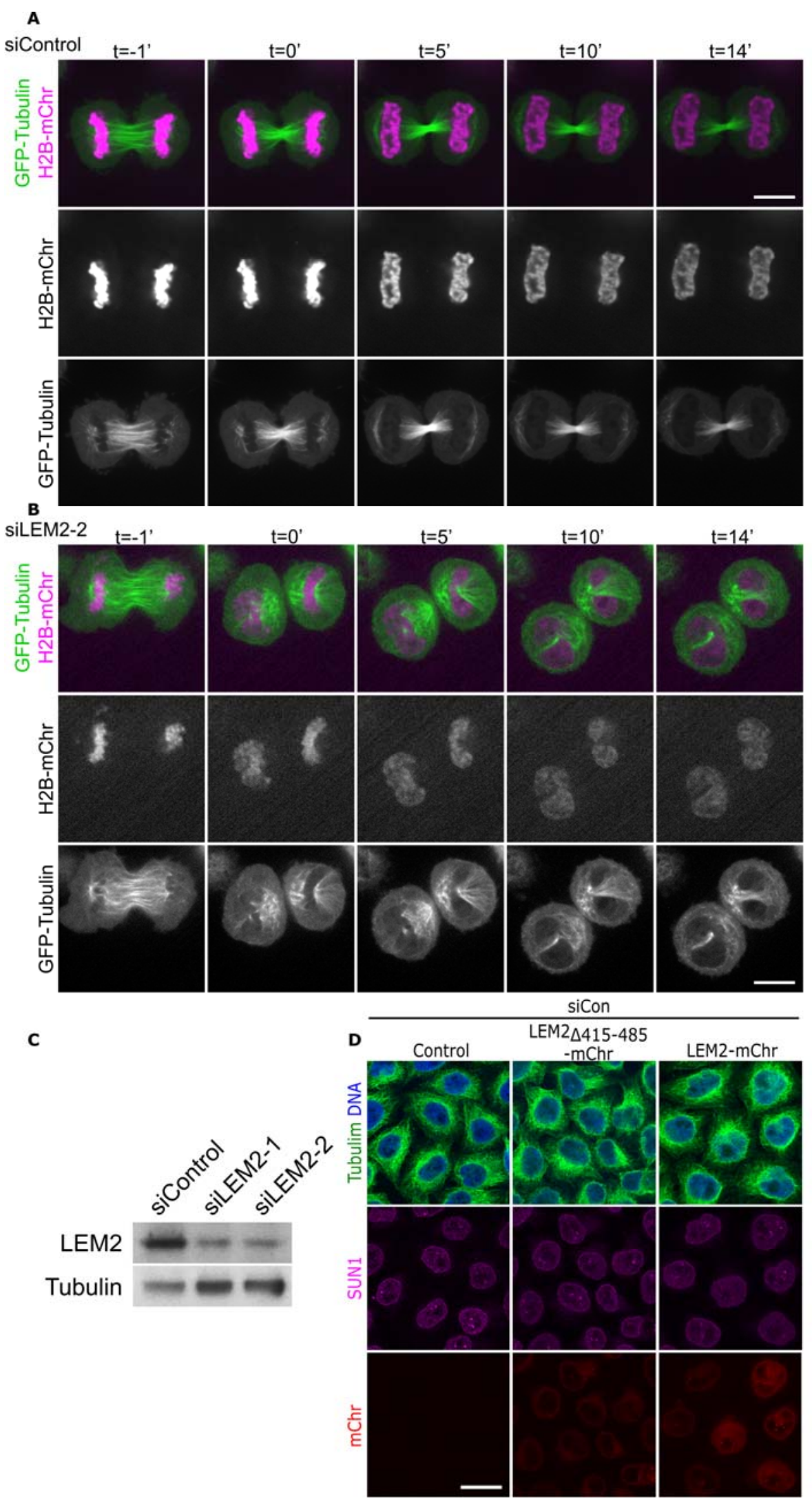


503 Extended Data Fig. 5. Development of post-mitotic tubulin phenotype with LEM2

504 depletion. (A-B) Live imaging through mitotic exit of HeLa cells stably expressing GFP-

505 Tubulin and H2B-mChr with siRNA control (A) or siLEM2 treatment (B). (C) Immunoblot

506 demonstrating LEM2 depletion by two independent siRNAs in U2OS cells. Scale bars 10 um.

507 (D) Immunostained HeLa cells stably expressing no or indicated siRNA-resistant constructs

508 were treated with control siRNA and imaged in interphase by spinning disk confocal

509 microscopy. Scale bar 20 um. 


\section{Methods}

\section{Cytoskeletal proteins}

Porcine brain Tubulin reagents were purchased from Cytoskeleton, Inc., including unpolymerized

514 Tubulin (T238P), unpolymerized, HiLyte-647-labeled Tubulin (TL670M), and pre-formed microtubules

515 (MT002).

Purification of His $_{6}$-SUMO-tagged proteins

All purified proteins in this study were expressed in a pCA528 vector (WISP08-174; DNASU Plasmid Repository) in BL21-(DE3)-RIPL Escherichia coli cells using an N-terminal His ${ }_{6}$-SUMO affinity tag as described previously ${ }^{11}$. All plasmids listed in Table S2. Expression cultures (3- to 4-liter) were grown in ZY-5052 rich auto-induction media containing kanamycin, shaking $(220 \mathrm{rpm})$ for 3 hours

521 at $37^{\circ} \mathrm{C}$, then overnight at $19^{\circ} \mathrm{C}$. Cells were harvested by centrifugation.

522 Purification of BAF: Purification of full length, human BAF protein (Uniprot ID O75531) was adapted 523 from a published protocol ${ }^{5}$. The purification was performed exactly as described, except His ${ }_{6}$-SUMO-

524 BAF was cleaved with His $_{6}$-Ulp1 protease (30 min, room temperature) to suit the use of the His ${ }_{6}-\mathrm{SUMO}$ 525 affinity tag. Ultimately, Superdex 75 16/60 fractions containing BAF dimer were pooled, concentrated, 526 and flash frozen in liquid nitrogen and stored as single use aliquots at $-80^{\circ} \mathrm{C}$.

527 Purification of LEM2 proteins: Protein constructs originating from the human LEMD2 protein (Uniprot

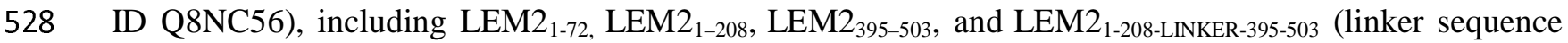
529 SAAGTGAGSGSAAS), LEM2 ${ }_{1-72}$, LEM$_{295-503}$, and LEM2 ${ }_{1-208-L I N K E R-395-503}$ constructs were purified as 530 follows. Harvested cells were resuspended in lysis buffer $(5 \mathrm{~mL}$ per gram cell pellet) containing $5 \%$ 531 glycerol, $10 \mathrm{mM}$ imidazole, $\mathrm{pH} 8.0,2 \mu \mathrm{g} / \mathrm{mL}$ DNase1, lysozyme and protease inhibitors, with HEPES and $532 \mathrm{KCl}$ concentrations optimized individually for each LEM2 protein construct (20 mM HEPES, pH 7.0, 500 $533 \mathrm{mM} \mathrm{KCl}$ for $\mathrm{LEM}_{1-72}$ and LEM2 ${ }_{1-208-L I N K E R-395-503}$, and $40 \mathrm{mM} \mathrm{HEPES}, \mathrm{pH} 8.0,350 \mathrm{mM} \mathrm{KCl}$ for 534 LEM2 $\left.{ }_{395-503}\right)$. Cells were lysed by sonication on ice, and clarified $\left(10,000 \mathrm{~g}, 30 \mathrm{~min}, 4^{\circ} \mathrm{C}\right)$. Clarified lysate 
was incubated with Ni-NTA agarose resin (Quiagen) ( $4 \mathrm{~mL}$ bed volume, $\left.1.5 \mathrm{~h}, 4^{\circ} \mathrm{C}\right)$, washed extensively with lysis buffer, and protein was eluted with 5 column volumes of lysis buffer supplemented with 350 $\mathrm{mM}$ imidazole, $\mathrm{pH}$ 8.0. Eluate was spin-concentrated (Vivaspin 20, 3KDa MWCO, PES) to $5 \mathrm{~mL}$, and dialyzed overnight at $4^{\circ} \mathrm{C}$ into storage buffer (20 - 40 mM HEPES, $\mathrm{pH} 7.0-8.0,5 \%$ glycerol), with $\mathrm{KCl}$ concentrations optimized individually for construct (300 mM for LEM2 $2_{1-72,} 500 \mathrm{mM}$ for LEM2 $2_{1-208-L I N K E R-}$ 395-503, and $350 \mathrm{mM}$ for LEM2 ${ }_{395-503}$ ). The $\mathrm{His}_{6}$-SUMO tag was cleaved by incubation with His ${ }_{6}$-Ulp1 protease during dialysis. LEM2 proteins were further purified by size exclusion chromatography using the Superdex 75 16/60 column (GE Life Sciences) in storage buffer, and LEM2-containing fractions were pooled, spin-concentrated (Vivaspin $63 \mathrm{KDa} \mathrm{MWCO}, \mathrm{PES}$ ), and aliquoted.

LEM2 $2_{1-208}$ was purified similarly, including phase separation as a preparative step. All steps were carried out at room temperature unless otherwise noted. Harvested cells were resuspended in lysis buffer (30 mM HEPES, pH 7.4, $500 \mathrm{mM} \mathrm{KCl,} \mathrm{5 \%} \mathrm{glycerol,} 10 \mathrm{mM}$ imidazole, $\mathrm{pH}$ 8.0, $2 \mu \mathrm{g} / \mathrm{mL}$ DNase1, lysozyme and protease inhibitors), and lysed by sonication with intermittent chilling on ice. Lysate was clarified by centrifugation $(10,000 \mathrm{~g}, 30 \mathrm{~min})$, incubated with Ni-NTA agarose resin ( $4 \mathrm{~mL}$ bed volume, $1.5 \mathrm{~h}$, room temperature), and washed extensively with lysis buffer. Protein was eluted with lysis buffer supplemented with $350 \mathrm{mM}$ imidazole, $\mathrm{pH}$ 8.0. Droplet formation was induced to enrich for $\mathrm{His}_{6}$-SUMOLEM2 $_{1-208}$ : the imidazole eluate was diluted with ice-cold buffer (40 mM HEPES, $\mathrm{pH} 7.4,5 \%$ glycerol) to drop the concentration of $\mathrm{KCl}$ to $50 \mathrm{mM}$. After $20 \mathrm{~min}$ incubation on ice, protein droplets were pelleted by centrifugation $\left(10,000 \mathrm{~g}, 10 \mathrm{~min}, 0^{\circ} \mathrm{C}\right)$, washed with 2 column volumes of ice-cold low-salt buffer (40 $\mathrm{mM}$ HEPES, $\mathrm{pH} 7.4,50 \mathrm{mM} \mathrm{KCl}, 5 \%$ glycerol), and pelleted again. The pellet was resuspended in $5 \mathrm{~mL}$ of high-salt buffer (40 mM HEPES, $\mathrm{pH} \mathrm{7.4,500} \mathrm{mM} \mathrm{KCl,} \mathrm{5 \%} \mathrm{glycerol),} \mathrm{to} \mathrm{dissolve} \mathrm{protein} \mathrm{droplets} \mathrm{for}$ the remaining purification steps, and incubated with $\mathrm{His}_{6}$-Ulp1 protease $(2 \mathrm{~h})$. The mixture was incubated with Ni-NTA resin $(2 \mathrm{~mL}, 1 \mathrm{~h})$. Spin-concentrated, flow-through protein was further purified by size exclusion chromatography using the Superdex $7516 / 60$ column in high-salt buffer at $4{ }^{\circ} \mathrm{C}$. Pooled, spin- 
concentrated, LEM2-containing fractions were dialyzed overnight into storage buffer (40 mM HEPES, pH 7.4, $350 \mathrm{mM} \mathrm{KCl,} \mathrm{5 \%} \mathrm{glycerol),} \mathrm{spin-concentrated,} \mathrm{and} \mathrm{aliquoted.}$

Concentrated protein aliquots were snap-frozen in liquid nitrogen and stored at $-80^{\circ} \mathrm{C}$ for future experiments. Aliquots were thawed and used only once. Final protein concentrations were $260 \mu \mathrm{M}$ for LEM2 $_{1-72}, \sim 110 \mu \mathrm{M}$ for LEM2 $2_{1-208}, 30 \mu \mathrm{M}$ for LEM2 $2_{1-208-L I N K E R-395-503}, 14 \mu \mathrm{M}$ for LEM2 $2_{395-503}$ and $190 \mu \mathrm{M}$ for His $_{6}$-SUMO-LEM2 ${ }_{395-503}$. Note that concentrations LEM2 $2_{395-503}$ without the tag were limited, and LEM2 $2_{395-503}$ was more stable with the $\mathrm{His}_{6}$-SUMO tag. and point mutants $\mathrm{CHMP} 7_{\mathrm{R} 270 \mathrm{E}}+\mathrm{I}_{132 \mathrm{E}}, \mathrm{CHMP} 7_{\mathrm{R} 270 \mathrm{E}}+\mathrm{V} 339 \mathrm{D}$, and $\mathrm{CHMP} 7_{\mathrm{I} 312 \mathrm{E}}+$ V339D were adapted from previously published purifications ${ }^{11}$. The truncated $\mathrm{CHMP}_{229-453}$ was purified differently. Harvested cells were resuspended in $5 \mathrm{~mL}$ ice-cold lysis buffer (40 mM HEPES, pH 8.0, $800 \mathrm{mM} \mathrm{KCl,} \mathrm{5 \%} \mathrm{glycerol,} 10$ $\mathrm{mM}$ imidazole, $2 \mu \mathrm{g} / \mathrm{mL}$ DNase1, $5 \mathrm{mM}$ BME, protease inhibitors, and lysozyme) per gram cell pellet.

571 Cells were lysed by sonication, on ice, and clarified $(10,000 \mathrm{~g}, 30 \mathrm{~min}$, room temperature). Clarified $572 \mathrm{CHMP}_{229-453}$ lysate was incubated with Ni-NTA agarose resin and spontaneously formed a gel composed 573 of protein polymerized into rings, assayed by negative stain EM. His 6 -SUMO-CHMP7 ${ }_{229-435}$ rings were 574 eluted with imidazole $(350 \mathrm{mM})$, and the eluate was collected with low-speed spin $(1,000 \mathrm{~g})$ as a gel phase 575 on top of the resin. The gel was scooped off, washed three times with buffer (40 mM HEPES, pH 8.0, 800 $576 \mathrm{mM} \mathrm{KCl}, 5 \%$ glycerol, $1 \mathrm{mM} \mathrm{DTT})$, and polymers were collected by centrifugation $(5,000 \mathrm{~g})$ each time. 577 The $\mathrm{His}_{6}$-SUMO tag was cleaved by incubation with $\mathrm{His}_{6}$-Ulp1 protease $\left(2 \mathrm{~h}, 4^{\circ} \mathrm{C}\right)$. Cleaved $\mathrm{CHMP}_{229-}$ 578453 was washed three times with buffer, collected by centrifugation each time, and soluble His ${ }_{6}-\mathrm{SUMO}$ 579 and $\mathrm{His}_{6}$-Ulp1 were discarded in the aqueous supernatant. Cleaved eluate was diluted with buffer to give 580 a final protein concentration of $\sim 60 \mu \mathrm{M}$. 
LEM2 ${ }_{1-72}$ binding to BAF or BAF-dsDNA complex was assayed by gel filtration chromatography. DNA duplex was prepared as previously described ${ }^{30}$. Combinations of purified LEM2 $2_{1-72}$, BAF dimers, and DNA duplexes were combined in 1:1:2 molar ratio. Following incubation at room temperature for 30 $\min , 50 \mu \mathrm{L}$ of reaction mixtures were applied to a Superose $63.2 / 300$ column (GE Life Sciences) in buffer (20 mM HEPES, pH 7.5, $150 \mathrm{mM} \mathrm{NaCl}, 5 \mathrm{mM}$ DTT, and $10 \%$ glycerol) equilibrated at $4^{\circ} \mathrm{C}$. The flow rate was $40 \mu \mathrm{L} / \mathrm{min}$ for all experiments. Retention volumes for major peaks absorbing at $280 \mathrm{~nm}$ (A280) were recorded. The protein contents of peak fractions were assayed by SDS-PAGE.

\section{LEM2 Low Complexity Domain Peptides}

Chemically synthesized peptides bearing an N-terminal FITC-Ahx modification (Figure S2C) were purchased from from GenScript (Piscataway, NJ), Peptide stock solutions were prepared in milli-Q H2O except LEM $2_{75-122}$ stocks, which were prepared in DMSO. Phase separation of LEM2 ${ }_{75-122}$ was induced by dilution in milli-Q $\mathrm{H} 2 \mathrm{O}$ or buffer (25 mM HEPES, pH 7.4, $150 \mathrm{mM} \mathrm{KCl}$ ) to $0.2 \mathrm{mg} / \mathrm{mL}$ stock.

\section{Turbidimetry}

Microtubule bundling by LEM2 was quantified by turbidity (absorbance at $340 \mathrm{~nm}$ ) (Tecan Spark synthesized LEM2 peptides, $1 \mu \mathrm{M} \alpha \beta$-Tubulin heterodimers polymerized in pre-formed microtubules, $\mathrm{KCl}$ (75 mM for LEM2 protein reactions, $0.9 \mathrm{mM}$ for LEM2 peptide reactions), $25 \mathrm{mM}$ HEPES, pH 7.4, $0.5 \mathrm{mM} \mathrm{MgCl} 2,10 \mu \mathrm{M}$ paclitaxel (Sigma Aldrich), were prepared at room temperature in 384-well non-

600 binding plates (Grendier Bio-One, \#781906). Turbidity was measured, with shaking before each read, for 601 reactions in quadruplicate, and averaged for each condition Turbidity values for reactions of LEM2 602 protein/peptide with microtubules were corrected for the turbidity of LEM2 protein/peptide alone, 603 normalized to turbidity of microtubules alone, and plotted (Turbidity a. u.) against [LEM2]. Sigmoidal 604 curve fitting and half-maximum LEM2 concentrations were calculated with GraphPad Prism software. 
605

606

607

608

609

610

611

612

613

614

615

616

617

618

619

620

621

622

623

624

625

626

627

Error bars are standard error of the mean. Turbidity data was corroborated by complementary negative stain electron microscopy.

\section{Protein labeling for fluorescence imaging}

Proteins were fluorescently labeled for microscopy using Microscale Protein Labeling kits from Thermo Scientific: Alexa Fluor ${ }^{\mathrm{TM}} 488$ (A30006), Alexa Fluor ${ }^{\mathrm{TM}} 555$ (A30007) and Alexa Fluor ${ }^{\mathrm{TM}} 647$ (A30009). LEM2 ${ }_{1-208}$ contains a single lysine residue and could not be efficiently labeled with primary amine reactive probes. Instead, His $_{6}-$ SUMO-LEM2 $2_{1-208}$ was labeled with Alexa- $488^{\mathrm{TM}}$ for fluorescence microscopy and a minimal amount was used to supplement indicated concentrations of unlabeled LEM $2_{1 \text { - }}$ 208 for experiments.

\section{Phase separation and droplet association assay}

LEM2 $2_{1-208}$ droplet formation was induced at room temperature by reducing the salt concentration to $150 \mathrm{mM} \mathrm{KCl}$, keeping the concentrations of other buffer components the same. Droplets were allowed to form and grow for $15 \mathrm{~min}$ before other components were added in tests for droplet association. Droplet association to MT was tested by the addition of $1 \mu \mathrm{M} \alpha \beta$-Tubulin polymerized into MT, following the Cytoskeleton, Inc. protocol, in a molar ratio of 1:7 HiLyte647-Tubulin:unlabeled Tubulin, in G-PEM buffer (Cytoskeleton Inc. BST01). Unpolymerized tubulin, 1:7 labeled:unlabeled molar ratio, and Alexa555-labeled LEM2 $2_{395-503}$ were tested for association with LEM2 $2_{1-208}$ droplets at concentrations of 8 $\mu \mathrm{M}$ each. Note that $150 \mathrm{mM} \mathrm{KCl}$ is prohibitive to polymerized tubulin in the absence of stabilizing proteins.

\section{Spinning Disk Confocal Microscopy with Fluorescence Recovery After Photobleaching (FRAP)}

Reactions were prepared in tubes and transferred into a glass-bottom 384-well plate (Grendier Bio-One, \#7781892) for imaging. LEM21-208 and LEM275-122 droplets were allowed to settle for 15 min before imaging. Spinning disk confocal microscopy with FRAP was carried out using a W1-CSU with a Borealis 

OptoElectronic) photobleaching unit and controlled though MicroManager 2.0beta (Open Imaging).

631 Fluorescence images were collected with an Andor Zyla 4.2 cMOS Camera (Oxford Instruments ).

632 Samples were imaged using a CFI Plan Apochromat VC 100X Oil NA 1.4 objective (Nikon Instruments,

633 Inc.). Excitation wavelengths were: $488 \mathrm{~nm}$ for Alex488TM or FITC; $561 \mathrm{~nm}$ for Alex555TM; $640 \mathrm{~nm}$

634 for Alex-647. Photobleaching for FRAP experiments was done with a $473 \mathrm{~nm}$ laser (Vortran) for $200 \mathrm{~ms}$

635 per bleach event. Images were recorded with a frequency of $1 \mathrm{~Hz}$, starting with at least one image

636 recorded pre-bleach, including the bleach event, and up to $240 \mathrm{~s}$ post-bleaching. Partial and whole droplet

637 FRAP experiments were performed in triplicate, and $n=17$ for the bundled-MT FRAP experiment. The

638 ratio of the intensity within bleached regions of interest to background were calculated with ImageJ for

639 each time point and normalized to the intensity at the time of bleaching. Replicates are aligned to the time

640 of bleaching, averaged, and plotted \pm standard deviation.

\section{Negative stain electron microscopy}

Continuous carbon grids (200-400 mesh copper, Quantifoil) were glow-discharged (PELCO

643 EasiGlow, $15 \mathrm{~mA}, 0.39 \mathrm{mBar}, 30 \mathrm{~s})$. Samples $(3-5 \mu \mathrm{L})$ were stained with $0.75 \%$ uranyl formate as

644 described previously ${ }^{25}$. Images were collected with a Tecnai T12 microscope (FEI Company, Hillsboro,

645 USA) with a LaB6 filament, operated at $120 \mathrm{kV}$, and data was captured with a Gatan Ultrascan CCD

646 camera (Gatan, Inc., Pleasanton, USA). For MT-binding assays, reactions were prepared on grids and

647 incubated for $2 \mathrm{~min}$. MT were used at a concentration of $1 \mu \mathrm{M} \alpha \beta$-Tubulin in pre-formed MT. LEM2 $2_{1-208}$

648 was screened for reactivity with MT at concentrations ranging from $0.25-4 \mu \mathrm{M}$, and LEM2 peptides

649 were screened at concentrations ranging from $10-200 \mu \mathrm{M}$. 
LEM2 and CHMP7 proteins were mixed at concentrations ranging from $4-8 \mu \mathrm{M}$ in a total volume of $50 \mu \mathrm{L}$. LEM2 ${ }_{395-503}$ was present in twofold molar excess to CHMP7 proteins, unless otherwise stated.

653 The buffer was adjusted to between 600 and $800 \mathrm{mM} \mathrm{KCl}$. Reactions were dialyzed for 9-12 h into low-

654 salt buffer (30 mM HEPES, pH 8.0, $25 \mathrm{mM} \mathrm{KCl,} \mathrm{and} 1 \mathrm{mM}$ DTT) using Slide-A-Lyzer MINI Dialysis

655 Device, 10K MWCO (Thermo Fisher Scientific). For experiments including MT, a 3x stock of pre-

656 formed, lyophilized microtubules was prepared in buffer (30 mM HEPES, pH 8.0, $800 \mathrm{mM} \mathrm{KCl,} 1 \mathrm{mM}$

657 DTT, $60 \mathrm{uM}$ paclitaxel, and $3 \mathrm{mM} \mathrm{MgCl}$ ), and dialysis buffer was supplemented with $10 \mu \mathrm{M}$ paclitaxel

658 and $1 \mathrm{mM} \mathrm{MgCl}$. After dialysis, polymeric assemblies were concentrated by low speed centrifugation

$659(5,000 \mathrm{~g}, 5 \mathrm{~min})$ and resuspended in $30 \mu \mathrm{L}$ buffer for negative stain EM, SDS-PAGE, or cross-linking mass spectrometry analysis. To determine the stoichiometry of the CHMP7-LEM2 ${ }_{395-503}$ polymer, CHMP7 $(4 \mu \mathrm{M})$ was titrated with $\operatorname{LEM}_{395-503}(0,0.08,1$ and $4 \mu \mathrm{M})$, dialyzed, and polymers wer

667 calculated, and error bars are standard deviation.

\section{Liposome preparation}

Lipid solutions (Avanti Polar Lipids) were resuspended in chloroform, and liposomes were

671 (mole \%) of 30\% egg phosphatidylserine (PS), 30\% egg phosphatidylcholine (PC) and 40\%

672 phosphatidylethanolamine (PE). Lipid films were dispersed in buffer (40 mM HEPES, pH 8.0, $150 \mathrm{mM}$

$673 \mathrm{KCl}$ ) to produce liposomes at a final concentration of $1 \mathrm{mg} / \mathrm{ml}$, and stored at $-80^{\circ} \mathrm{C}$. 
676 (supplemented with 5\% glycerol, $40 \mathrm{mM}$ HEPES, pH 8.0, $1 \mathrm{mM}$ DTT) to give a final protein 677 concentration of $0.1 \mathrm{mg} / \mathrm{ml}$. Liposomes and CHMP7 were mixed 40:1 (w/w) and negative stain EM grids 678 were prepared immediately.

Electron Microscopy data acquisition and 2D classification orientation for subsequent classification. Particles were picked and 2D-classified with RELION software.

\section{Cross-linking Mass spectrometry} concentration. Full length, monomeric CHMP7 $(60 \mu \mathrm{g})$ was crosslinked with $2 \mathrm{mM}$ heavy crosslinker ( $\mathrm{D}_{12}$-BS3, Creative Molecules) under the same conditions. Reactions were quenched (10 mM ammonium bicarbonate, $10 \mathrm{~min}$, room temperature), and light and heavy crosslinked reaction mixtures were

694 combined and processed for mass spectrometry as described previously ${ }^{33,34}$. Crosslinked products were 695 enriched by size-exclusion chromatography (Superdex Peptide, GE Healthcare Life Sciences) as 696 described previously ${ }^{34}$ and fractions eluting between 0.9 and $1.4 \mathrm{~mL}$ were dried, resuspended in $0.1 \%$ 697 formic acid for MS analysis. The fractions starting at $0.9 \mathrm{ml}$ and $1.3 \mathrm{ml}$ were combined prior to 698 evaporation to make four total SEC fractions. 

acetonitrile). Precursor MS scans were measured in the Orbitrap scanning from $350-1500 \mathrm{~m} / \mathrm{z}$ (mass resolution: 70,000). The ten most intense triply charged or higher precursors were isolated in the quadrupole (isolation window: $4 \mathrm{~m} / \mathrm{z}$ ), dissociated by HCD (normalized collision energy: 24), and the product ion spectra were measured in the Orbitrap (mass resolution: 17,500). A dynamic exclusion window of $20 \mathrm{sec}$ was applied and the automatic gain control targets were set to $3 \mathrm{e} 6$ (precursor scan) and 5e4 (product scan). searched for with Protein Prospector 5.20.23 ${ }^{35} .85$ of the most intense peaks from each product ion spectrum were searched against a database containing His $_{6}$-SUMO-LEM2 ${ }_{395-503}$ along with the sequences of 10 other proteins comprising CHMP subunits and Tubulin, concatenated with 10 randomized decoy versions of each of these sequences (121 sequences total). Search parameters were: mass tolerance of 20 ppm (precursor) and $30 \mathrm{ppm}$ (product); fixed modifications of carbamidomethylation on cysteine; variable modifications of peptide N-terminal glutamine conversion to pyroglutamate, oxidation of methionine, and "dead-end" modification of lysine and the protein N-terminus by semi-hydrolyzed heavy

717 and light BS3; trypsin specificity was used with 2 missed cleavages and three variable modifications per 718 peptide were allowed. Unique, crosslinked residue pairs were reported at a $1.5 \%$ FDR threshold, 719 corresponding to a Score Difference cutoff of 15. 
724 paired with its corresponding heavy or light counterpart. Precursor ion transitions matching the first three

725 isotopes were extracted across all four LC-MS fractions. Each extracted ion chromatogram was manually

726 inspected and the start and end points were adjusted to ensure that the correct peaks were detected and

727 that there were no interfering signals. Isotope dot products were required to exceed 0.85 . The peak areas

728 were re-imported into $\mathrm{R}$ and summarized at the level of crosslinked residues for each light and heavy

729 crosslink. Peak areas were summed for all peptides matching a given crosslink. Finally, $\log 2$ ratios of the

730 heavy to light peak areas were determined. Filtered cross-links were mapped to the primary protein

731 structure using xiNET ${ }^{38}$. Quantitative changes are displayed as a cross-link contact map using an in house

732 R-package. The $\mathrm{x}-\mathrm{y}$ - axes map to protein and residue position, with each dot representing a cross-linked

733 pair. The area of the dot is proportional to the number of spectral counts identifying a cross-link (total

734 number of product ion spectra that match a cross-linked residue pair), while enrichment in either

735 monomeric (heavy BS3 - red) or polymeric (light BS3 - blue) sample is indicated by the color scale.

\section{Homology modeling and cross-link mapping}

Homology models of human CHMP7 and LEM2 domains were created with Phyre2 ${ }^{39}$ (Table S1).

Reference structures were selected based on confidence scores and homology to reference structure.

Xlink analyzer ${ }^{41}$.

\section{Immunostaining for fluorescence microscopy}

HeLa cells were fixed at room temperature in $2 \%$ paraformaldehyde for $25 \mathrm{~min}$. The primary

743 antibodies used for immunodetection were rabbit $\alpha$-IST1 ${ }^{42}$, rabbit $\alpha$-Tubulin (ab18251; abcam), rat $\alpha$ -

744 Tubulin (YL1/2; Accurate Chemical \& Scientific), SUN1 (ab124770; abcam), SUN2 (gift from Brian

745 Burke), and mouse $\alpha-53 B P 1$ (MAB3802; Millipore). After incubation with fluorescently labeled

746 secondary antibodies ( $\alpha$-rabbit 488, $\alpha$-mouse 488, $\alpha$-rabbit 647, and $\alpha$-rabbit 568; Thermo Fisher),

747 coverslips were mounted with DAPI ProLong Gold (Thermo Fisher) and imaged by either widefield 
748

749

750

751

752

753

754

755

756

757

758

759

760

761

762

763

microscopy (Zeiss Axioskop 2; Axiovision software), spinning disk confocal microscopy (Nikon Eclipse $\mathrm{T} i$; Metamorph software), or stimulated emission depletion microscopy. For quantifying IST1 localization: $\alpha$-rabbit 488 against rabbit $\alpha$-IST1; for assessing DNA Damage: $\alpha$-mouse 488 against mouse $\alpha$-53BP1 and $\alpha$-rabbit 568 against rabbit $\alpha$-Tubulin; for quantifying the Tubulin and nuclear envelope phenotype: $\alpha$-mouse 488 against mouse $\alpha$-SUN2 and $\alpha$-rabbit 568 against rabbit $\alpha$-Tubulin; for interphase phenotype: $\alpha$-rabbit 647 against rabbit $\alpha$-SUN1 and $\alpha$-mouse 488 against rat $\alpha$-Tubulin; for STED: $\alpha$-mouse 568 against mouse $\alpha$-Tubulin and $\alpha$-rabbit 647 against rabbit $\alpha$-LEM2.

\section{Stimulated Emission Depletion (STED) microscopy}

STED microscopy was performed on a Leica TCS SP8 STED 3X confocal laser-scanning microscope equipped with a HC PL APO CS2 100x/1.40 OIL objective. Confocal sections were imaged with Leica LAS X Core software and processed with Huygens Software Suite (SVI). Images were recorded using $405 \mathrm{~nm}$ laser line at $1.4 \%$ laser power to image DAPI, and a $572 \mathrm{~nm}$ Laser line at $5.6 \%$ laser power to image Tubulin in confocal detector mode. LEM2 was imaged with a $653 \mathrm{~nm}$ laser line at $2.5 \%$ laser power in STED pulsed detector mode (gate start at $0.3 \mathrm{~ns}$ and gate end at $6.5 \mathrm{~ns}$ ) with a Huygens saturation factor of 5.7. Deconvolved Images were further processed in ImageJ (NIH).

\section{Statistics}

For time-lapse colocalization experiments tracking GFP-CHMP7 with either LEM2-mChr or

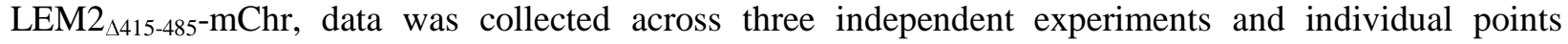
corresponding to each cell (two disks) were plotted showing standard deviation (LEM2-mChr: $n=14$; LEM $2_{\triangle 415-485}-\mathrm{mChr}: n=11$; see below for method of quantification). For quantification of IST1 recruitment, the mean percent of anaphase disks for each category of IST1 recruitment was determined from three independent experiments and plotted showing standard error of the mean (see below for method of quantification; sample size $(n)$ and raw data shown in Supplementary Table 1). For quantification of nuclear accumulation of NLS-3xGFP, data was collected across three independent 
772

773

774

775

776

777

778

779

780

781

782

783

experiments and the mean plotted showing standard deviation (siControl LEM2-mChr: $n=18$; siLEM2-2 LEM2-mChr: $n=14$; siControl LEM2 $2_{\triangle 415-485}-\mathrm{mChr}: n=24$; siControl LEM2 $2_{\Delta 415-485}-\mathrm{mChr}$ : $n=6$; see below for method of quantification). For quantification of NE-tubulin defects, the mean percent of telophase cells with NE-tubulin defects was determined from three independent experiments and plotted showing standard error of the mean (siControl: $n=75,35,37$; siLEM2-1: $n=72,35,37$; siLEM2-2: $n=45,34,34$ ). For quantification of DNA damage, the mean percent of telophase cells with $\geq 553 \mathrm{BP} 1$ foci was determined from three independent experiments and plotted showing standard error of the mean (siControl: $n=56,52,44$; siLEM2-1: $n=64,56,26$; siLEM2-2: $n=74,50,40$ ). In all cases, two-tailed unpaired t-test was used to determine p-values, which, unless otherwise specified, are indicated as follows: ${ }^{*} P<0.05 ; * * P<0.01 ; * * * P<0.001 ;$ N.S., not significant.

\section{Quantification from fluorescence microscopy}

For illustration, images of anaphase A and B cells were acquired by widefield microscopy at $100 \mathrm{X}$ and adjusted so that background fluorescence in the DNA, IST1, and LEM2-mChr channels were comparable between samples. Raw images acquired by widefield microscopy at $100 \mathrm{X}$ were used to score the IST1 phenotype in anaphase A (early) and anaphase B (late). IST1 localization to anaphase chromatin masses was assessed in three independent experiments in which images were randomized and quantified blindly by three independent scorers. Each chromatin disk (two per cell) was scored as having extra robust, robust, weak, or no recruitment for IST1. Robust recruitment was characterized by distinctive foci organized at the core of chromatin masses, whereas weak recruitment was characterized by less intense, often fewer, and less organized foci at the chromatin surface, consistent with what has previously been shown $^{11}$. Extra robust was characterized by strikingly intense IST1 fluorescence, often accompanied by recruitment over most of the chromatin disk. The majority score was used in cases where the three scores differed. 
For time-lapse colocalization experiments tracking GFP-CHMP7, images of anaphase cells were acquired by spinning disk confocal microscopy at $60 \mathrm{X}$ and were selected for scoring at the time of peak LEM2-mCherry or LEM $2_{\Delta 415-485}$-mCherry enrichment at the nuclear envelope. In ImageJ, each cell was thresholded for either LEM2-mCherry or LEM2 ${ }_{\triangle 415-485}$-mCherry enrichment. The mean fluorescence (arbitrary units) was measured for each region of interest in the GFP-CHMP7 channel. The regions of interest were subtracted from the area of the whole cell to measure the mean fluorescence of cytoplasmic

802 GFP-CHMP7 for each anaphase cell. The plotted values are the mean GFP-CHMP7 fluorescence of the 803 region of interest, as determined by exogenous LEM2 enrichment, normalized to the cytoplasmic levels of 804 GFP-CHMP7. Plotted points are for individual cells, which were imaged across three different experiments.

Nuclear accumulation of NLS-3xGFP was determined by taking the ratio of nuclear to cytoplasmic NLS-3xGFP at different time points throughout anaphase following imaging by spinning disk confocal microscopy at $60 \mathrm{X}$. In ImageJ, regions of chromatin were defined as regions of interest in the NucBlue ${ }^{\mathrm{TM}}$ channel and used to measure the mean fluorescence (arbitrary units) in the NLS-3xGFP channel. Cytoplasmic levels of NLS-3xGFP were determined by selecting the whole cell, and subsequently 811 deselecting the regions of chromatin. The mean fluorescence of nuclear NLS-3xGFP for each disk was 812 then divided by the mean fluorescence of cytoplasmic NLS-3xGFP of the same cell. Data was collected 813 across three independent experiments and plotted showing standard deviation.

For the purposes of illustration, interphase and telophase cells were acquired by widefield and spinning disk confocal microscopy at $60 \mathrm{X}$ and were adjusted so that background fluorescence was comparable between samples. Raw images used to score the nuclear envelope and Tubulin phenotype

817 were acquired by widefield microscopy at $60 \mathrm{X}$ in three independent experiments. To score DNA damage, 818 images from three independent experiments were acquired by widefield microscopy at $60 \mathrm{X}$ and and noise tolerance was held constant for all conditions. 


\section{Time-Lapse Light Microscopy Analysis}

822

823

824

825

826

827

828

829

830

831

832

833

834

835

836

837

838

839

840

841

842

843

For all live imaging experiments images were acquired at $60 \mathrm{X}$ and complete cleavage furrow ingression is designated as $\mathrm{t}=0$ minutes.

Stable cell lines used in this study are described in Table S2. Cells stably expressing GFP-Tubulin ${ }^{43}$ were transiently transfected with siRNA resistant pCMV $(\Delta 5)$-LEM2-mCherry (PL13), pCMV $(\Delta 5)$ LEM2m21-mCherry $\quad(\mathrm{PL} 14), \quad \operatorname{pCMV}(\Delta 5)-\mathrm{LEM} 2_{\triangle 43-202}$-mCherry $\quad(\mathrm{PL} 15), \quad \operatorname{pCMV}(\Delta 5)-\mathrm{LEM} 2_{\triangle 145-213^{-}}$ mCherry (PL16), or pCMV( $\triangle 5$ )-LEM2 $2_{\triangle 415-485}$-mCherry (PL17) using Lipofectamine LTX Plus (Thermo Fisher) for $24 \mathrm{~h}$. Cells were then re-plated on Mat-Tek dishes $8 \mathrm{~h}$ before being arrested at G1/S and released, as described below. Twelve hours after release, cells were live-imaged by spinning disk confocal microscopy in the presence of NucBlue ${ }^{\mathrm{TM}}$ (Thermo Fisher).

For time-lapse colocalization experiments, HeLa cells stably expressing GFP-CHMP7 incubated overnight. and transiently transfected with either siRNA resistant pCMV( $\triangle 5)$-LEM2-mCherry (PL13) or siRNA resistant pCMV $(\Delta 5)$-LEM2 ${ }_{\triangle 415-485}$-mCherry (PL17) using Lipofectamine LTX Plus (Thermo Fisher) for $24 \mathrm{~h}$. Cells were then re-plated on Mat-Tek dishes $8 \mathrm{~h}$ before being arrested at G1/S and released, as described below. Twelve hours after release, cells were live-imaged by spinning disk confocal microscopy.

To track nuclear integrity, cells stably expressing NLS-3xGFP (PL19) and either siRNA resistant $\operatorname{pCMV}(\triangle 5)-L E M 2-m C h e r r y(P L 13)$ or $\operatorname{pCMV}(\triangle 5)-L E M 2_{\triangle 415-485}-\mathrm{mCherry}(\mathrm{PL} 17)$ were plated on MatTek dishes in the presence of siRNA $8 \mathrm{~h}$ before being arrested at G1/S and released, as described below. Twelve hours after release, cells were live-imaged by spinning disk confocal microscopy in the presence of NucBlue $\mathrm{TM}^{\mathrm{TM}}$ (Thermo Fisher) in 15 second intervals.

For siRNA depletion in HeLa cells stably expressing H2B-mCherry and GFP-Tubulin, cells were plated on fibronectin coated Mat-Tek dishes in the presence of siRNA $8 \mathrm{~h}$ before being arrested at G1/S 
844 and released, as described below. Twelve hours after release, cells were live-imaged by spinning disk

845 confocal microscopy.

Cells stably expressing GFP-BAF and LEM2-mCherry were plated on fibronectin coated Mat-Tek

847 dishes $8 \mathrm{~h}$ before being arrested at G1/S and released, as described below. Twelve hours after release,

848 cells were live-imaged by spinning disk confocal microscopy.

siRNA-Mediated Depletion and Cell-Cycle Synchronization

HeLa and U2OS cells were plated on fibronectin-coated coverslips in the presence of $10 \mathrm{nM}$ siRNA

oligo, delivered by Lipofectamine RNAiMAX Transfection Reagent (Thermo Fisher). Specific sequences

852 used were: siControl [siScr-1 ${ }^{43,44}$ ], siLEM2-1 [antisense sequence targeting nucleotides 78-98:

853 UUGCGGUAGACAUCCCGGGdTdT ${ }^{27}$ ], and siLEM2-2 [antisense sequence targeting nucleotides

854 1,297-1,317: UACAUAUGGAUAGCGCUCCdTdT ${ }^{27}$ ]. Culture medium containing $2 \mathrm{mM}$ thymidine was

855 then added for $24 \mathrm{~h}$ to arrest cells at G1/S. Cells were then rinsed thoroughly with PBS, followed by the

856 addition of culture media. Twelve hours after release, cells were imaged live or fixed for microscopy.

\section{Immunoblots}

To verify efficacy of siRNA treatments and expression of siRNA-resistant constructs, cells were

859 plated in six-well dishes and subjected to the same experimental conditions as those used to generate data.

860 Whole cell lysates were prepared with NP40 lysis buffer, diluted in SDS sample buffer supplemented

861 with DTT, and boiled. Samples were run on 10\% SDS-PAGE gels and transferred to PVDF membrane.

$8623 \%$ milk in TBS-T was used for blocking and antibody dilutions. After incubation with primary

863 antibodies [ $\alpha$-LEM2 (HPA017340; Sigma-Aldrich) and $\alpha$-Tubulin (ab18251; abcam)], reactivity was

864 detected with HRP-coupled secondary antibodies (Thermo Fisher) and chemiluminescence. 
866 Extended Data Table 1. Template Structures Used for Homology Modeling.

\begin{tabular}{|c|c|c|c|c|}
\hline Protein & $\begin{array}{l}\text { AAs covered by } \\
\text { model }\end{array}$ & $\begin{array}{l}\text { Template } \\
\text { Structure }\end{array}$ & $\begin{array}{l}\text { Model } \\
\text { Organism }\end{array}$ & $\begin{array}{l}\text { PDB } \\
\text { Identifier }\end{array}$ \\
\hline CHMP7 $_{\text {ESCRTIII }}$ open & $238-356$ & SNF7 & S.c. & 5FD7 (23) \\
\hline $\begin{array}{l}\text { CHMP7 ESCRTIII } \\
\text { closed }\end{array}$ & $235-373$ & CHMP3 & H.s. & 2GD5 (51) \\
\hline $\mathrm{CHMP}_{\mathrm{WH} 1}+\mathrm{WH}_{2}$ & $42-223$ & VPS25 & H.S. & 3CUQ (52) \\
\hline $\mathrm{LEM}_{\mathrm{WH}}$ & $396-501$ & MAN1 & H.s. & $2 \mathrm{CH} 0(20)$ \\
\hline
\end{tabular}

867

868 
Extended Data Table 2. Plasmids and cell lines used in this study.

871 transfecting parental HeLa cells with the described plasmids using Lipofectamine LTX Plus

872 (Thermo Fisher) for $24 \mathrm{~h}$ before selection with the specified antibiotics.

\begin{tabular}{|c|c|c|c|c|c|}
\hline \multicolumn{6}{|c|}{ Plasmids } \\
\hline ID & Construct & Vector and Source & Protein & Tag & $\begin{array}{l}\text { Expression } \\
\text { System }\end{array}$ \\
\hline PL1 & $\mathrm{CHMP}_{229-453}$ & pCA528 & CHMP7 & $\mathrm{His}_{6}-\mathrm{SUMO}$ & E. coli \\
\hline PL2 & CHMP7 & pCA528 & CHMP7 & $\mathrm{His}_{6}-\mathrm{SUMO}$ & E. coli \\
\hline PL3 & $\mathrm{CHMP}_{\mathrm{R} 270 \mathrm{E}}+\mathrm{I312E}$ & pCA528 & CHMP7 & His $_{6}-\mathrm{SUMO}$ & E. coli \\
\hline PL4 & $\mathrm{CHMP}_{\mathrm{R} 270 \mathrm{E}+\mathrm{V} 339 \mathrm{D}}$ & pCA528 & CHMP7 & His $_{6}-\mathrm{SUMO}$ & E. coli \\
\hline PL5 & CHMP7I312E + V339D & pCA528 & CHMP7 & His $_{6}-\mathrm{SUMO}$ & E. coli \\
\hline PL6 & LEM2 $_{1-72}$ & pCA528 & LEM2 & $\mathrm{His}_{6}-\mathrm{SUMO}$ & E. coli \\
\hline PL7 & LEM2 $2_{1-208}$ & pCA528 & LEM2 & His $_{6}-\mathrm{SUMO}$ & E. coli \\
\hline PL8 & LEM2 $395-503$ & pCA528 & LEM2 & His $_{6}-\mathrm{SUMO}$ & E. coli \\
\hline PL10 & LEM21-208-LINKER-395-503 & pCA528 & LEM2 & His $_{6}-\mathrm{SUMO}$ & E. coli \\
\hline PL11 & $\mathrm{BAF}$ & pCA528 & BAF & $\mathrm{His}_{6}-\mathrm{SUMO}$ & E. coli \\
\hline PL12 & $\begin{array}{l}\text { siRNA resistant } \\
\text { GFP-CHMP7 }\end{array}$ & From pMGF182 (8) & CHMP7 & GFP & Mammalian \\
\hline PL13 & $\begin{array}{l}\text { siRNA resistant } \\
\text { LEM2-mCherry }\end{array}$ & From pMGF196 (8) & LEM2 & mCherry & Mammalian \\
\hline PL14 & $\begin{array}{l}\text { siRNA resistant } \\
\text { LEM } 2 \mathrm{~m} 21-\mathrm{mCherry}\end{array}$ & From PL13 & LEM2 & mCherry & Mammalian \\
\hline PL15 & $\begin{array}{l}\text { siRNA resistant } \\
\text { LEM } 2_{\triangle 43-202} \text {-mCherry }\end{array}$ & From PL13 & LEM2 & mCherry & Mammalian \\
\hline PL16 & $\begin{array}{l}\text { siRNA resistant } \\
\text { LEM2 } 2_{\Delta 145-213-m C h e r r y}\end{array}$ & From PL13 & LEM2 & mCherry & Mammalian \\
\hline PL17 & $\begin{array}{l}\text { siRNA resistant } \\
\text { LEM2 } 2_{\Delta 415-485-m C h e r r y}\end{array}$ & From PL13 & LEM2 & mCherry & Mammalian \\
\hline PL18 & eGFP-BAF & $\begin{array}{l}\text { From pLX304 } \\
\text { (from DNASU) and } \\
\text { EGFP-C1 backbone }\end{array}$ & BAF & GFP & Mammalian \\
\hline PL19 & NLS-3xGFP & $\begin{array}{l}\text { Gift from Martin } \\
\text { Hetzer }\end{array}$ & $\begin{array}{l}\text { NLS- } \\
3 \times G F P\end{array}$ & $\begin{array}{l}\text { NLS fused to } \\
\text { triple GFP }\end{array}$ & Mammalian \\
\hline Stable & Cell Lines & & & & \\
\hline Expre & ed constructs & Plasmid used & $\begin{array}{l}\text { Parental } \\
\text { cell line }\end{array}$ & Selection & \\
\hline GFP-1 & abulin + H2B-mCherry & Generated previo & asly (49) & G418, Puro & nycin \\
\hline GFP-C & HMP7 & PL12 & HeLa & G418 & \\
\hline LEM2 & $\mathrm{mChr}$ & PL13 & HeLa & Puromycin & \\
\hline
\end{tabular}




\begin{tabular}{|l|l|l|l|}
\hline LEM2 ${ }_{\Delta 415-485-m C h r}$ & PL17 & HeLa & Puromycin \\
\hline GFP-BAF + LEM2-mChr & PL13 and PL18 & HeLa & G418, Puromycin \\
\hline NLS-3xGFP and LEM2-mChr & PL19 and PL13 & HeLa & G418, Puromycin \\
\hline $\begin{array}{l}\text { NLS-3xGFP and LEM2 } \\
\text { mChr }\end{array}$ & PL15-485- and PL17 & HeLa & G418, Puromycin \\
\hline
\end{tabular}

874 Extended Data Movie 1. LEM2 droplet formation.

875 Droplet formation of $32 \mathrm{uM} \mathrm{LEM} 2_{1-208}$ was induced by reducing the salt concentration from 500 $\mathrm{mM}$ to $150 \mathrm{mM} \mathrm{KCl}$ in presence of $0.5 \mathrm{uM}$ Alexa488 labeled His ${ }_{6}-\mathrm{SUMO}-\mathrm{LEM}_{1-208}$. Sample volume was $40 \mu 1$. Images were recorded every min for 120 min total.

Extended Data Movie 2. Droplet fusion.

Film clip from ED Movie 1 highlighting

881

Extended Data Movie 3. A series of Z-stack images illustrative of the LEM2-depletion

\section{phenotype in telophase cells.}

HeLa cells stably expressing H2B-mCherry and GFP-Tubulin were treated with control or

LEM2-targeting siRNA and live imaged by spinning disk confocal microscopy. absent for IST1. 


\section{Supplementary Table 2. Quantified Cross-links from comparative mass spectrometry}

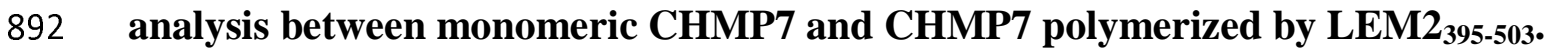

893 Summed peak areas for identified cross-links are listed for Heavy (polymeric CHMP7) and Light

894 (Monomeric CHMP7). In case the identified cross-link was absent in Light sample, the value was set to

89540000 for analysis purposes and the $\log 2$ ratio is shown as n/a. Cross-links with $\log 2$ ratios between 2 and

$896-2$ as well as cross-links falling into the region of the His6-SUMO-tag present in LEM2 $2_{395-503}$ were filtered

897 and not considered in the analysis.

898 